\title{
Evolution and Traditional Importance of Manda National Park (MNP) in the Moyen-Chari Province of CHAD
}

\author{
Esaie Waya ${ }^{1,2,{ }^{*}}$, Ibrahima Adamou ${ }^{1}$, Tchoua Paul ${ }^{3}$ \\ ${ }^{1}$ Department of Biological Sciences, University of Ngaoundere, Ngaoundere, Cameroon \\ ${ }^{2}$ Department of Environment Sciences, University of Sarh, Sarh, Chad \\ ${ }^{3}$ Departement of Mathematical, University of Ngaoundere, Ngaoundere, Cameroun
}

\author{
Email address: \\ wayason@yahoo.fr (E. Waya) \\ ${ }^{*}$ Corresponding author
}

\section{To cite this article:}

Esaie Waya, Ibrahima Adamou, Tchoua Paul. Evolution and Traditional Importance of Manda National Park (MNP) in the Moyen-Chari Province of CHAD. International Journal of Natural Resource Ecology and Management. Vol. 6, No. 2, 2021, pp. 65-78.

doi: 10.11648/j.ijnrem.20210602.16

Received: April 23, 2021; Accepted: May 13, 2021; Published: May 31, 2021

\begin{abstract}
The study aims to analyze the traditional perception of riparian populations on the spatial-temporal evolution and importance of the Manda National Park (MNP) in the Moyen-Chari Province, in order to contribute to the implementation of a participatory and efficient strategy for its sustainable management. To do this, a survey was conducted using a questionnaire with 211 people composed of forestry officials and non-officials from the four (04) townships bordering the park. The data collected concerned the evolution of the vegetation cover, uses, degradation of plant resources and their causes. The results show that the MNP has suffered from anthropic pressure, resulting in a regression of the vegetation cover in a global way from 2009 to 2019. The main causes of the decrease in vegetation cover are overgrazing, poaching, bush fires, logging and hunting. The main uses of MNP's forest resources are for food, fodder, energy fuel, and traditional medicine. The plant species most used for various purposes are Anogeissus leiocarpus (42.36\%), Terminalia laxiflora (26.97\%), Flueggea virosa (21.3\%), Detarium microcarpum (20.11\%) and Vitellaria paradoxa (19.70\%). The four townships do not differ significantly from each other in the types of uses of forest resources and the causes of their degradation. The results of scientific studies obtained on the dynamics of the park's vegetation cover confirm traditional perceptions. Hence the importance of involving local populations in studies on the sustainable management of the park.
\end{abstract}

Keywords: Survey, Spatiotemporal Evolution, Perception, Population, Vegetation, Management, MNP and Chad

\section{Introduction}

The network of protected areas in Chad covers nearly $10.2 \%$ of the country's surface area and remains globally representative of all the diversity of ecosystems in the region [1]. Protected areas are defined as "a geographically defined area of land, water or marine environment especially dedicated to the protection and maintenance of biological diversity and associated natural and cultural resources; for these purposes this geographical area must be legally designated, regulated and administered by effective legal and other means" [2]. The conservation and sustainable management of national parks, which belong to the domain of protected areas, allows for the maintenance of various ecological functions such as production, protection, and social and cultural values [3]. The Manda National Park (MNP) located in southeastern Chad and classified as a national park in 1965, is one of the last refuges of wildlife in the Moyen-Chari. Its globally important biodiversity has experienced periods of relative calm as well as significant disturbances due to anthropic pressure and climatic deterioration [4] and poor political and institutional conditions (4th National Report, 2009). This anthropic pressure is manifested through pastoral activities, increasingly organized poaching, late bush fires and agriculture at the edge of the park. In addition, poor 
institutional policy is manifested through frequent bribery and corruption, not to mention the poverty of the riparian population and growing insecurity $[5,6]$. To counteract its degradation, UNDP, UNOPS and GEF have funded a project called "Project for the conservation and sustainable use of biodiversity in the Moyen-Chari"to address its conservation. For this, detailed knowledge of its traditional importance, its structure, the factors of degradation of its phytodiversity and its dynamics that depend on pedoclimatic and anthropic conditions is paramount $[7,8]$. In Chad in general and particularly in the Moyen-Chari province, very little information exists on $\operatorname{MNP}[5,6,9,10]$. The objective of this study is to assess the traditional socioeconomic importance of Manda National Park (MNP) and its vegetation dynamics in order to contribute to its sustainable management in the context of current anthropogenic pressure.

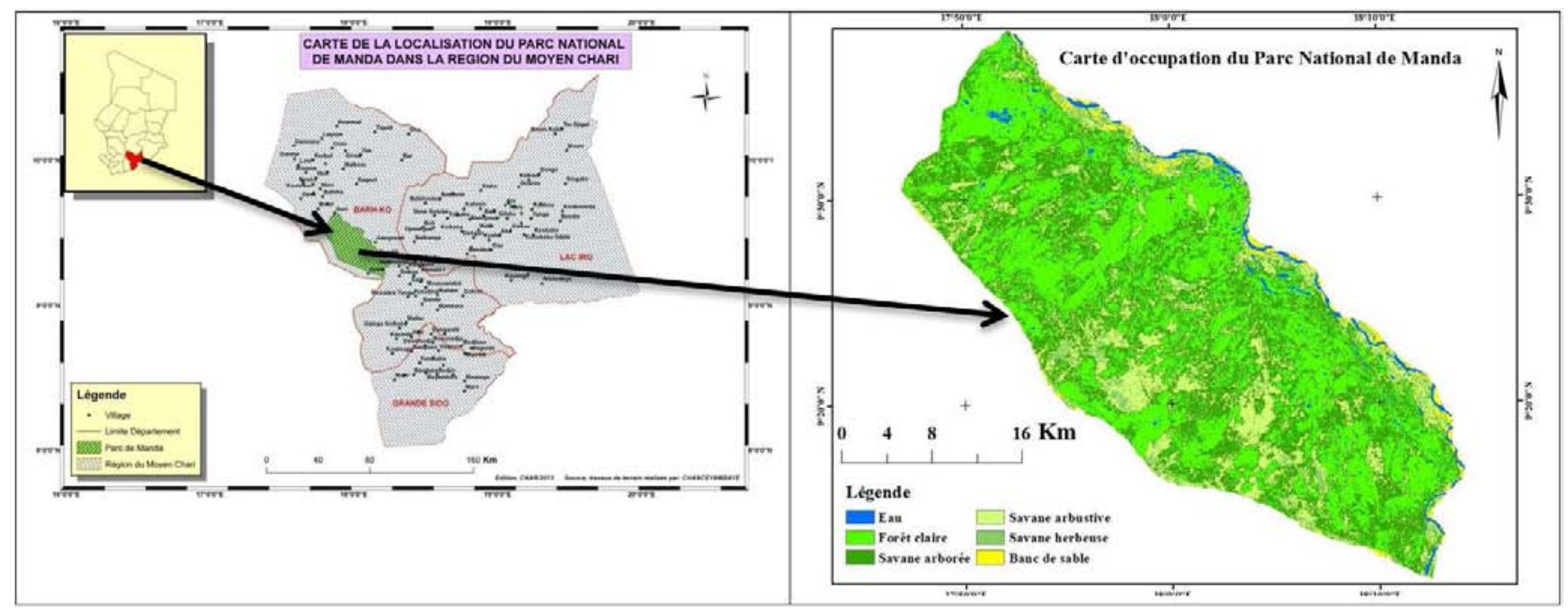

Figure 1. Map of Manda National Park (Source: Waya E., 2019). Materials and Methods.

\section{Material and Methods}

\subsection{Study Site}

The study sites are located in the Manda National Park (MNP), which is $25 \mathrm{~km}$ northwest of Sarh, capital of the Barh-Kôh Department, Moyen-Chari Province (Figure 1). It lies between $9^{\circ} 20^{\prime}$ and $9^{\circ} 35^{\prime}$ north latitude and $17^{\circ} 45^{\prime}$ and $18^{\circ} 20^{\prime}$ east longitude. Its altitude varies from 344 to $691 \mathrm{~m}$. The area is characterized by a Sudanese type of climate with an average annual rainfall of $1061.41 \mathrm{~mm}$. The temperature varies between 21 to $28^{\circ} \mathrm{C}$ with an annual average of $24.5^{\circ} \mathrm{C}$ and a relative humidity depending on the month of 32 to $85 \%$ [11]. The soils are varied, the following main types are encountered Erosion soils on acidic rocks that are dominant on Mount Niellim; sesquioxides with ferruginous stains and concretions and cuirasses that are found on lateritic slabs in the Niellim zone; vertisols in certain flood zones between Koutou and Niellim; Ferralitic soils that are widely exposed in the Koutou and Djoli zones; tropical ferruginous soils in the center of the park, from Nguéré to Koutou; and hydromorphic soils characteristic of soils in the south of the park [12]. The MNP is served by a hydrographic network, in its eastern part by the Chari River and in the south by the Barh Sara. Two of the three main tributaries of the Chari have their confluences in the MNP: the Bahr Sara and the Bahr Salamat. In addition, 13 temporary and 23 permanent pools have been identified in the park [13]. The vegetation formations are gallery forests, shrub and tree savannahs. On the whole, the vegetation is of the Sudanian type, the density and distribution of which are a function of the topography and the nature of the soil. Outside the plain, open forest and wooded savannah with dominant legumes alternate with patches of dry forest or riparian forest [14]. The diversity of wildlife is underestimated. Only vertebrates can be considered moderately studied, with about ten wild families reported by UICN/PACO [6]. The peripheral zone surrounding the MNP includes 85592 inhabitants distributed in four townships: Balimba in the south, Kokaga in the east, Djoli in the west and Niellim in the north [15]. The peripheral population of the MNP is mainly composed of farmers. Fishing activities mainly concern the riparian population of the rivers and fishermen. The lands bordering the park also constitute a reception area for an increasing number of pastoralists. According to the survey conducted by Ballah [1], the sedentary livestock population is estimated at 28.843 head of cattle and 13.789 head of goats. The annual rate of ruminant livestock is estimated by the Animal Resources and Livestock Directorate (DERA) at 2.4\% per year. Four townships bordering the MNP were selected to conduct the surveys. These are the townships of Balimba, Djoli, Kokaga and Niellim. They differ in ethnicity and religion. Around the MNP, we find the Sar Madjingaye in Balimba Township and part of Djoli Township; the Sara-Kaba and Tounia in Kokaga Township; the Niellim and Boua in Niellim Township. Finally, the Toumak and Ndam dominate in Djoli Township. The population is predominantly Christian 
or animist in Balimba and Djoli, while in Kokaga and Niellim townships it is Muslim or animist.

\subsection{Methodology}

The study was carried out in households, on site and with township leaders and forestry services, in the form of a survey using a questionnaire in the four (04) townships bordering the MNP during the period from January to March 2020. The type of interview used is the so-called semistructured one including closed questions for which one answer yes or no, open questions for which one answer deliberately according to one's point of view and guided questions for which some answers are proposed at the beginning. The sample consisted of 211 people, including 200 local residents, four cantonal chiefs, four chiefs of the cantonal forestry service and three provincial administrators from the Ministry of the Environment, Water and Fisheries. The main headings of the questionnaire concerned the socioeconomic importance of the park, the traditional perception of the spatial and temporal evolution of the MNP, and finally the traditional strategies for sustainable management of the MNP. The individual interviews were facilitated by interpreters who spoke the local dialects (Sara, Niellim, Boua Arabic and Tounia). The species cited during the survey were determined from their local names, then collected and identified with the help of botanists from the University of Sarh, botanical lexicons [15], and reference materials (Adventrop, 1995; ligneux du sahel, 2008, Arbonnier, 2009; Flore illustrée du Tchad, 2019).

\subsection{Processing of Survey Data}

The approach taken is easily adapted to the requirements of our research. It is done in two stages: the processing and the computerized handling of the data collected in the field. The first step, which involved the processing of quantitative and qualitative data, consisted of verifying that the questionnaires, had been filled out and that the answers given by the respondents corresponded. Generally speaking, this involves carefully examining result sheets to extract information. The second stage is marked by the manipulation of the data carried out. This phase required the use of computer tools. The descriptive analyses were carried out using Microsoft Office Excel 2010, to determine the averages and percentages of the different characteristics of the respondents, their activities, their length of time in the localities surrounding the MNP, their modes of taking natural resources in the MNP; the different forms of anthropic pressure exerted on the MNP and the different uses of plant species in the MNP. It is a treatment that takes into account qualitative data and quantitative data.

\section{Results}

\subsection{Characteristics of Respondents}

Table 1 summarizes the characteristics of the respondents by township. There are more men (148) than women (52), i.e. $74 \%$ for men and $26 \%$ for women. The male gender represents more than half of the total number of respondents in the different townships. In each of the 4 townships, men outnumbered women. The age range of the respondents varies from 25 to over 56 years old. The majority of respondents are on average between 25 and 40 years old (39\% of responses), and in Balimba (44\%) and Djoli (36\%) townships. In contrast, in Kokaga Township, the majority of respondents are between 40 and 55 years old (42\%). People over 55 years of age are less numerous in the samples. On average, it is estimated at $24.5 \%$, or $34 \%$ in Djoli, 22\% in Balimba and Kokaga, and $20 \%$ in Niellim. The majority of respondents, regardless of sex, are married (90\%), i.e., $80 \%$ in Niellim, $49 \%$ in Kokaga, $47 \%$ in Balimba, and $44 \%$ in Djoli, followed by widowers $(6.5 \%)$, bachelors $(2 \%)$, and finally divorcees $(1.5 \%)$. The majority of respondents have primary education with an average of $41.5 \%$. This rate is $52 \%$ in Balimba and Djoli, $34 \%$ in Kokaga and $28 \%$ in Niellim, followed by the non-literate (37\%), i.e. $54 \%$ in Kokaga, $50 \%$ in Niellim, $30 \%$ in Balimba and $14 \%$ in Djoli, few of them have reached secondary level $(20.5 \%)$, i.e. $34 \%$ in Djoli, $20 \%$ in Niellim, $16 \%$ in Balimba and $12 \%$ in Kokaga, and finally, those who have a higher level of education are almost non-existent They are found only in the townships of Balimba and Niellim, with $2 \%$ of responses for each.

Table 1. Characteristics of respondents in the four (04) townships (responses in \%).

\begin{tabular}{|c|c|c|c|c|c|c|}
\hline \multirow{2}{*}{ Variables } & \multirow{2}{*}{ Modalités } & \multicolumn{4}{|c|}{ Townschips } & \multirow{2}{*}{ Average } \\
\hline & & Balimba & Djoli & Kokaga & Niellim & \\
\hline \multirow{2}{*}{ Genre } & Male & 76 & 76 & 68 & 76 & 74 \\
\hline & Female & 24 & 24 & 32 & 24 & 26 \\
\hline \multirow{3}{*}{ Age rang } & {$[25-40]$} & 44 & 36 & 36 & 40 & 39 \\
\hline & [40-55] & 34 & 30 & 42 & 40 & 36.5 \\
\hline & $>55$ & 22 & 34 & 22 & 20 & 24.5 \\
\hline \multirow{4}{*}{ Status } & Married & 47 & 44 & 49 & 80 & 90 \\
\hline & Widowed & 2 & 4 & 0 & 14 & 6.5 \\
\hline & Single & 1 & 1 & 1 & 2 & 2 \\
\hline & Divorced & 0 & 1 & 0 & 4 & 1.5 \\
\hline \multirow{4}{*}{ Education } & Non literate & 30 & 14 & 54 & 50 & 37 \\
\hline & Primary & 52 & 52 & 34 & 28 & 41.5 \\
\hline & Secondary & 16 & 34 & 12 & 20 & 20.5 \\
\hline & Higher & 2 & 0 & 0 & 2 & 1 \\
\hline
\end{tabular}




\subsection{Activities of the People Living Near the MNP}

The activities of people living along the shores of MNP are diverse and varied (Figure 2). However, combined activities such as agro-livestock farming and agro-fishing are the most dominant, with average responses of $42.5 \%$ and $20 \%$ respectively for agro-livestock farming and agro-fishing. These two main activities are followed by agriculture practiced by $24 \%$ of the populations. Agribusiness, fishing and other activities are practiced very little in the four townships, with less than $9 \%$ of respondents responding. By township, while agro-breeding dominates in Djoli $(74 \%)$ and Niellim (44\%), agro-fishing comes first in Kokaga (42\%) and Balimba (24\%). These two main activities are followed by agriculture, which is practiced by $24 \%$ of the population in Niellim, 14\% in Balimba and Djoli, and $8 \%$ in Kokaga.

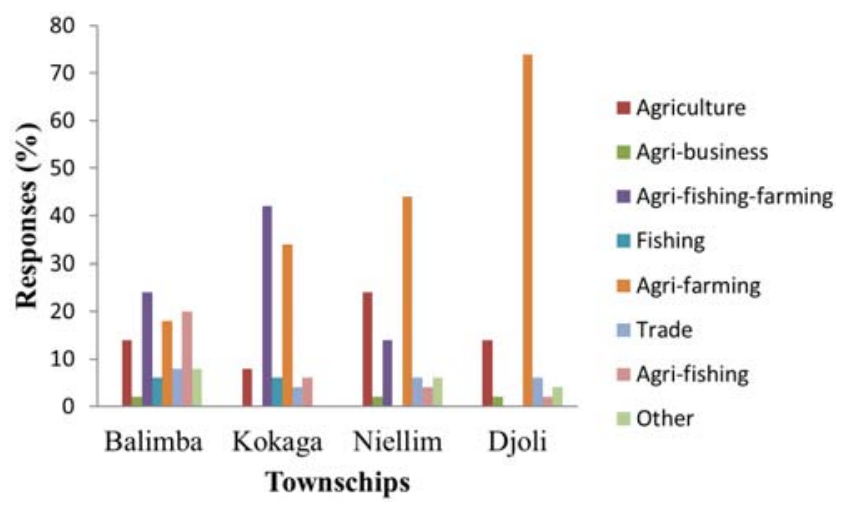

Figure 2. Activities of MNP residents.

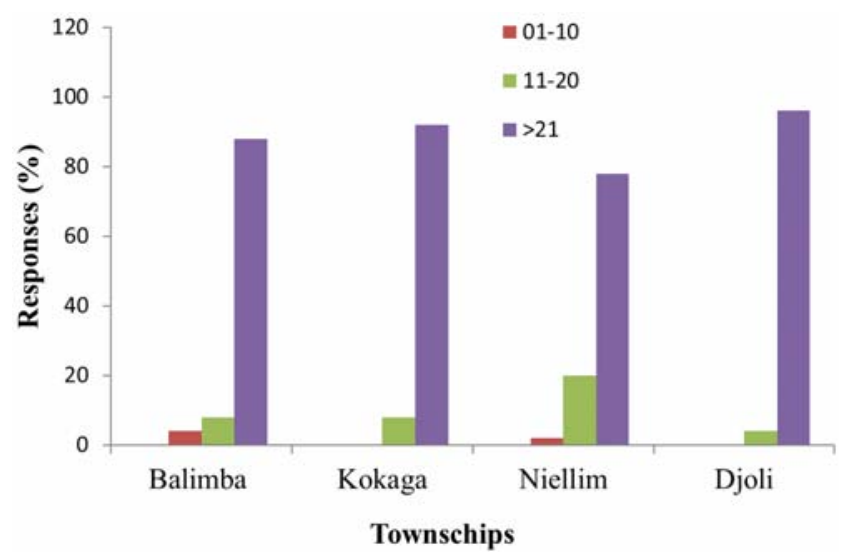

Figure 3. Seniority in the localities surrounding the MNP.

Most of these respondents have been living in the localities bordering the MNP for more than 21 years (Figure 3). Those under 11 years of age are very poorly represented in the townships, and even absent in Kokaga and Djoli townships.

\subsection{Indicators of Anthropogenic Pressures}

The results of the survey indicate a high level of anthropogenic pressure that can negatively affect the park's vegetation (Figure 4). The main indicators of anthropogenic pressure cited by local residents are grazing (33.45\%), poaching $(30.62 \%)$, bush fires $(18.52 \%)$, and harvesting of timber forest products (LFPs) on average (15.87\%). The harvesting of non-timber forest products (NTFPs) is low $(1.51 \%)$. In the four townships, the main indicators of anthropic pressure are grazing and poaching, with respective response rates of 39\% in Djoli, 33.85\% in Niellim, $29.68 \%$ in Balimba and $23.43 \%$ in Kokaga for grazing and $32.31 \%$ in Niellim, $30.51 \%$ in Djoli, $28.38 \%$ in Balimba and $22.86 \%$ in Kokaga for poaching. The weakest indicators cited by local residents are the harvesting of non-timber forest products (NTFPs), with response rates of $9.68 \%$ in Balimba, $6.78 \%$ in Djoli and $0.37 \%$ in Niellim, except in Kokaga where NTFPs are the least cited (16.57\%). Finally, bushfire and LFPs are moderately cited, except in Kokaga where bushfire and NTFPs are moderately cited.

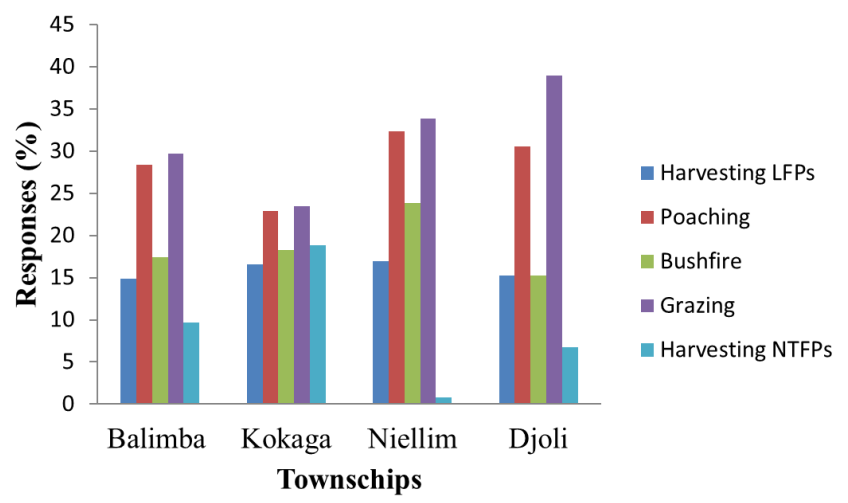

Figure 4. Different forms of anthropogenic pressures on the MNP.

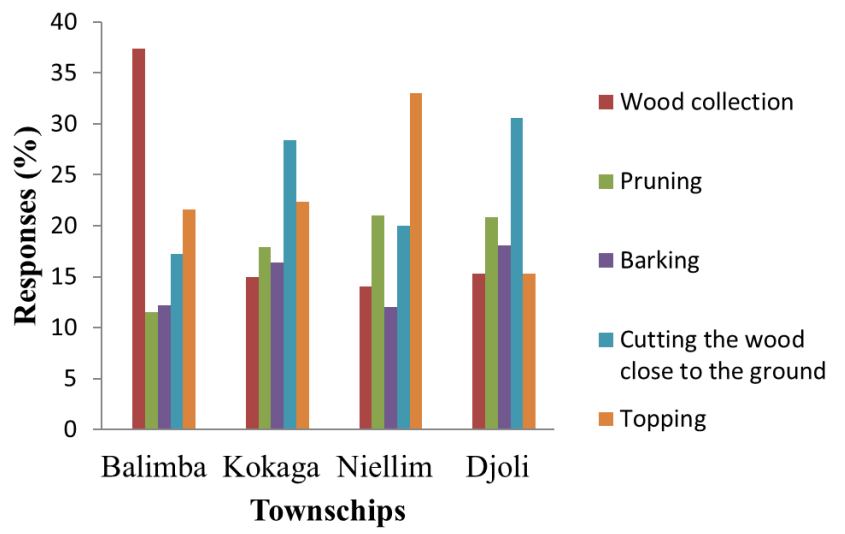

Figure 5. Patterns of natural resource harvesting in MNP.

The method of harvesting forest resources is collecting, pruning, debarking, flush cutting and topping (Figure 5). The main methods used are flush cutting (24.05\%), topping $(23.06 \%)$, and gathering $(20.41 \%)$, and the least cited is debarking (14.68\%). Other harvesting methods such as pruning were moderately cited $(16.93 \%)$. These harvesting methods vary between townships. While in Balimba, collecting is the highest $(37.41 \%)$ and debarking and pruning are the lowest with respective response rates of 12.23 and $11.51 \%$, in Niellim, it is rather topping that is most practiced $(33.00 \%)$ and the least practiced is debarking (12\%). As for the other two townships, Kokaga and Djoli, cutting wood is 
the most used method with response rates of $28.36 \%$ and $30.56 \%$ respectively, and the least used is collecting with response rates of $14.92 \%$ and $15.28 \%$ respectively. In addition, topping is also the least practiced activity at the same level as gathering, with each $15.28 \%$ response.

\subsection{Evolution of MNP Vegetation Between 2009 and 2019}

Almost all of the people surveyed (92\%) claimed to know the park's boundaries and are therefore able to assess the evolution of its vegetation, in particular its area, density and the causes of this evolution. According to the responses of MNP riparian residents, three states of MNP vegetation were determined between 2009 and 2019, namely regression, stagnation and progression (Table 2). The majority of the riparians $(46.25 \%)$ claim that there is regression of MNP vegetation during the period from 2009 to 2019 . Those who think, that is increasing $(36.03 \%)$ come second, while those who see stagnation of MNP vegetation are low, with $17.71 \%$ of respondents. The area and density of vegetation are higher according to respondents who think that the vegetation is in a state of progression, with responses of 47.14 and $45.66 \%$ respectively. The percentage of responses from respondents who think that the PNM is in a state of stagnation is low, with respective responses of 19.01 and $14.29 \%$ for the area and density.

Table 2. Farmers' responses (\%) of vegetation change and its causes between 2009 and 2019.

\begin{tabular}{|c|c|c|c|c|}
\hline \multirow{2}{*}{$\begin{array}{l}\text { Vegetation } \\
\text { Status }\end{array}$} & \multicolumn{3}{|c|}{ 2009-2019 } & \multirow[b]{2}{*}{ Causes } \\
\hline & Total & Area & Density & \\
\hline Declining & 46,25 & 47,14 & 45,66 & $\begin{array}{l}\text { Climate change, winds insufficient number of forestry agents, overgrazing, poaching, poor management, late } \\
\text { bush fires, logging, laxity of the mobile brigade }\end{array}$ \\
\hline Stagnant & 17,71 & 19,01 & 14,29 & Less logging, good surveillance by forestry agents and fear of entering the MNP because of its prohibition \\
\hline Increasing & 36,03 & 33,84 & 37,50 & $\begin{array}{l}\text { its prohibition, good surveillance by forestry agents } \\
\text { Contribution of species, fertilization of the soil by the presence of domestic animal dung and wildlife }\end{array}$ \\
\hline
\end{tabular}

\subsection{Types of Use of Forest Resources Harvested in Manda National Park}

Forest resources from MNP's phytodiversity are used in a variety of ways by local people in the four townships, particularly for food, traditional medicines, and as a source of fodder, energy, and building materials (Table 3). Overall, forest resources are widely used by the riparian populations of MNP (100\%). Between cantons, the populations of Niellim use MNP's natural resources less (11-18\%) than those of the other three cantons (Balimba, Kokaga and Djoli), with response rates ranging from 19-36\%.

The types of use vary among the townships (Table 3 ). In fact, in Niellim Township, the population uses more MNP forest resources for fuel energy (18.14\%) than for other types of use. On the other hand, in Balimba Township, forest resources are used more for fodder $(32.42 \%)$. In Kokaga Township, these forest resources are used for construction materials (34.39\%) and finally, in Djoli Township, these forest resources are used for food $(28.2 \%)$ and traditional medicine (28.20\%). Globally, the main species that contribute the most to the plant resources of MNP used by the riparians are Anogeissus leiocarpus (42.36\%), Terminalia laxiflora (26.97\%), Flueggea virosa (21.3\%), Detarium microcarpum (20.11\%), Vitellaria paradoxa (19.70\%), Propsopis africana (18.66\%), Combretum collinum (17.43\%), Mitragyna inermis (17.07\%), Hymenocardia acida (15.83\%), Piliostigma thonningii (14.85\%), Parkia biglobosa (12.92\%), Vitex doniana (12.00\%), Ziziphus abyssinica (11.42\%) and Afzelia africana (10.12\%). Between townships, Parkia biglobosa is the most used species in food in Balimba $(3.42 \%)$, Detarium microcarpum in Djoli $(10.8 \%)$ and Vitelleria paradoxa (4.16\%) in both Balimba (4.16\%) and Djoli (3.42\%). Species such as Flueggea virosa (7.98\%), Trephrosia deflaxa (4.47\%) and Afzelia africana (3.5\%) are the most popular species for cattle in Kokaga, Balimba and Djoli townships respectively. The most popular species for timber in each of the townships are Prosopis africana $(4.26 \%)$ and Anogeissus leiocarpus (3.88\%) in Djoli, Mitragyna inermis (3.97\%) and Anogeissus leiocarpus (3.88\%) in Balimba and Kokaga respectively. Of these species, Anogeissus leiocarpus (6.53\%) and Combretum collinum $(4.13 \%)$ in Djoli and Terminalia laxiflora (4.00\%) in Balimba are the species par excellence for firewood. The most popular species for traditional pharmacopoeia in Balimba, Djoli and Kokaga are Terminalia laxiflora (2.66\%), Hyminocardia acida (2.66\%) and Anogeissus leiocarpus $(2.04 \%)$ respectively.

Table 3. MNP forest products used by local residents for socio-economic purposes (\% responses).

\begin{tabular}{|c|c|c|c|c|c|c|c|c|c|c|c|c|c|c|c|}
\hline \multirow{2}{*}{ Espèces } & \multicolumn{5}{|c|}{ Feeding } & \multicolumn{5}{|c|}{ Fodder } & \multicolumn{5}{|c|}{ Service } \\
\hline & $\mathbf{A}$ & B & $\mathbf{C}$ & D & $\mathbf{T}$ & $\mathbf{A}$ & B & $\mathbf{C}$ & D & $\mathbf{T}$ & $\mathbf{A}$ & B & $\mathbf{C}$ & D & $\mathbf{T}$ \\
\hline Acacia sieberiana & 0,00 & 0,00 & 0,00 & 0,00 & 0 & 0,00 & 0,39 & 0,58 & 0,00 & 0,97 & 0,00 & 0,00 & 0,00 & 0,00 & 0 \\
\hline Afzelia africana & 0,09 & 0,00 & 0,00 & 0,09 & 0,18 & 2,53 & 1,56 & 0,58 & 3,50 & 8,17 & 0,00 & 0,09 & 0,28 & 0,00 & 0,37 \\
\hline Albizia adianthifolia & 0,00 & 0,00 & 0,00 & 0,00 & 0 & 0,00 & 0,00 & 0,00 & 0,00 & 0 & 0,00 & 0,00 & 0,00 & 0,00 & 0 \\
\hline Allophylus africanus & 0,00 & 0,00 & 0,00 & 0,00 & 0 & 0,00 & 0,00 & 0,00 & 0,00 & 0 & 0,00 & 0,00 & 0,09 & 0,00 & 0,09 \\
\hline Amblygonocarpus andongensis & 0,00 & 0,00 & 0,00 & 0,09 & 0,09 & 0,00 & 0,19 & 0,19 & 0,00 & 0,38 & 0,00 & 0,00 & 0,00 & 0,28 & 0,28 \\
\hline Anchomanes difformis & 0,00 & 0,00 & 0,00 & 0,00 & 0 & 0,00 & 0,00 & 0,00 & 0,00 & 0 & 0,00 & 0,00 & 0,00 & 0,00 & 0 \\
\hline Andropogon gayanus & 0,00 & 0,00 & 0,00 & 0,00 & 0 & 0,00 & 0,00 & 0,00 & 0,00 & 0 & 0,00 & 0,00 & 0,00 & 0,38 & 0,38 \\
\hline
\end{tabular}




\begin{tabular}{|c|c|c|c|c|c|c|c|c|c|c|c|c|c|c|c|}
\hline \multirow{2}{*}{ Espèces } & \multicolumn{5}{|c|}{ Feeding } & \multicolumn{5}{|c|}{ Fodder } & \multicolumn{5}{|c|}{ Service } \\
\hline & $\mathbf{A}$ & B & $\mathbf{C}$ & D & $\mathbf{T}$ & $\mathbf{A}$ & $\mathbf{B}$ & $\mathbf{C}$ & D & $\mathbf{T}$ & $\mathbf{A}$ & B & $\mathbf{C}$ & D & $\mathbf{T}$ \\
\hline Annona senegalensis & 0,00 & 0,92 & 0,18 & 0,09 & 1,19 & 0,78 & 0,58 & 0,19 & 0,78 & 2,33 & 0,00 & 0,38 & 0,09 & 0,00 & 0,47 \\
\hline Anogeissus leiocarpa & 0,00 & 0,00 & 0,00 & 0,00 & 0 & 1,17 & 0,00 & 1,95 & 0,78 & 3,9 & 2,93 & 2,75 & 3,88 & 3,88 & 13,44 \\
\hline Balanites aegyptiaca & 0,46 & 0,18 & 2,49 & 0,46 & 3,59 & 0,00 & 0,19 & 0,19 & 0,00 & 0,38 & 0,28 & 0,00 & 0,00 & 0,00 & 0,28 \\
\hline Bauhinia rufescens & 0,00 & 0,00 & 0,00 & 0,00 & 0 & 0,00 & 0,00 & 0,00 & 0,00 & 0 & 0,00 & 0,00 & 0,00 & 0,00 & 0 \\
\hline Boerhavia erecta & 0,00 & 0,00 & 0,00 & 0,00 & 0 & 0,00 & 0,19 & 0,00 & 0,00 & 0,19 & 0,00 & 0,00 & 0,00 & 0,00 & 0 \\
\hline Borassus aethiopum & 0,55 & 0,18 & 0,18 & 0,18 & 1,09 & 0,00 & 0,00 & 0,00 & 0,00 & 0 & 0,38 & 0,00 & 0,00 & 0,00 & 0,38 \\
\hline Bridelia ferruginea & 0,00 & 0,00 & 0,00 & 0,00 & 0 & 0,00 & 2,53 & 0,39 & 1,75 & 4,67 & 0,00 & 0,09 & 0,00 & 0,00 & 0,09 \\
\hline Burkea africana & 0,00 & 0,00 & 0,00 & 0,00 & 0 & 0,00 & 0,19 & 0,00 & 0,00 & 0,19 & 0,00 & 0,00 & 0,00 & 0,76 & 0,76 \\
\hline Capparis sepiaria var fischeri & 0,00 & 0,00 & 0,00 & 0,00 & 0 & 0,00 & 0,00 & 0,00 & 0,00 & 0 & 0,00 & 0,00 & 0,00 & 0,00 & 0 \\
\hline Cassia occidentalis & 0,00 & 0,00 & 0,00 & 0,00 & 0 & 0,00 & 0,00 & 0,00 & 0,00 & 0 & 0,00 & 0,00 & 0,00 & 0,00 & 0 \\
\hline Cassia sieberiana & 0,00 & 0,00 & 0,09 & 0,00 & 0,09 & 0,00 & 0,19 & 0,58 & 0,00 & 0,77 & 0,00 & 0,28 & 0,19 & 0,00 & 0,47 \\
\hline Celtis integrifolia & 0,00 & 0,00 & 0,00 & 0,00 & 0 & 0,00 & 0,19 & 1,17 & 0,00 & 1,36 & 0,00 & 0,00 & 0,00 & 0,00 & 0 \\
\hline Cissus populnea & 0,00 & 0,00 & 0,00 & 0,00 & 0 & 0,00 & 0,19 & 0,00 & 0,00 & 0,19 & 0,00 & 0,19 & 0,57 & 0,00 & 0,76 \\
\hline Combretum collinum & 0,00 & 0,09 & 0,00 & 0,00 & 0,09 & 0,00 & 0,39 & 0,58 & 0,00 & 0,97 & 0,09 & 0,00 & 0,76 & 0,19 & 1,04 \\
\hline Combretum glutinosum & 0,00 & 0,09 & 0,00 & 0,00 & 0,09 & 0,00 & 0,00 & 0,00 & 0,19 & 0,19 & 0,00 & 0,00 & 0,09 & 0,00 & 0,09 \\
\hline Combretum molle & 0,00 & 0,00 & 0,00 & 0,00 & 0,00 & 0,00 & 0,00 & 0,00 & 0,00 & 0 & 0,00 & 0,38 & 0,00 & 0,00 & 0,38 \\
\hline Combretum nigricans & 0,00 & 0,00 & 0,00 & 0,00 & 0,00 & 0,00 & 0,00 & 0,00 & 0,19 & 0,19 & 0,00 & 0,09 & 0,00 & 0,00 & 0,09 \\
\hline Commiphora pedunculata & 0,00 & 0,00 & 0,00 & 0,00 & 0,00 & 0,00 & 0,00 & 0,00 & 0,00 & 0 & 0,00 & 0,00 & 0,00 & 0,00 & 0 \\
\hline Cordia africana & 0,00 & 0,09 & 0,00 & 0,00 & 0,09 & 0,00 & 0,00 & 0,00 & 0,00 & 0 & 0,00 & 0,00 & 0,00 & 0,00 & 0 \\
\hline Crossopteryx febrifuga & 0,00 & 0,09 & 0,09 & 0,00 & 0,18 & 0,00 & 0,00 & 0,00 & 0,00 & 0 & 0,00 & 0,38 & 0,00 & 0,00 & 0,38 \\
\hline Cymbopogon giganteus & 0,00 & 0,00 & 0,00 & 0,00 & 0,00 & 0,00 & 0,00 & 0,00 & 0,00 & 0 & 0,00 & 0,09 & 0,00 & 0,09 & 0,18 \\
\hline Daniellia oliveri & 0,00 & 0,09 & 0,00 & 0,00 & 0,09 & 0,78 & 0,00 & 0,19 & 0,19 & 1,16 & 0,00 & 0,00 & 0,00 & 0,00 & 0 \\
\hline Detarium microcarpum & 1,94 & 2,95 & 2,31 & 3,60 & 10,80 & 0,00 & 0,00 & 0,00 & 0,19 & 0,19 & 0,66 & 0,00 & 0,00 & 0,09 & 0,75 \\
\hline Dioscorea dumetorum & 0,18 & 0,09 & 0,18 & 0,55 & 1,00 & 0,00 & 0,00 & 0,00 & 0,00 & 0 & 0,00 & 0,00 & 0,00 & 0,00 & 0 \\
\hline Diospyros mespiliformis & 0,09 & 1,29 & 0,65 & 0,28 & 2,31 & 0,00 & 0,39 & 0,19 & 0,00 & 0,58 & 0,00 & 0,47 & 0,09 & 0,00 & 0,56 \\
\hline Ectadiopsis oblongifolia & 0,00 & 0,00 & 0,00 & 0,00 & 0,00 & 0,00 & 0,19 & 0,00 & 0,39 & 0,58 & 0,00 & 0,00 & 0,00 & 0,19 & 0,19 \\
\hline Entada africana & 0,00 & 0,00 & 0,00 & 0,00 & 0,00 & 0,00 & 0,58 & 0,00 & 0,00 & 0,58 & 0,00 & 0,00 & 0,00 & 0,00 & 0 \\
\hline Eragrostis atrovirens & 0,00 & 0,00 & 0,00 & 0,00 & 0,00 & 0,00 & 0,00 & 0,00 & 0,00 & 0 & 0,00 & 0,00 & 0,00 & 0,00 & 0 \\
\hline Eragrostis ciliaris & 0,00 & 0,00 & 0,00 & 0,00 & 0,00 & 0,00 & 0,00 & 0,00 & 0,00 & 0 & 0,00 & 0,19 & 0,28 & 0,00 & 0,47 \\
\hline Euphorbia kamerunica & 0,18 & 0,00 & 0,00 & 0,00 & 0,18 & 0,00 & 0,00 & 0,00 & 0,00 & 0 & 0,09 & 0,00 & 0,00 & 0,00 & 0,09 \\
\hline Faidherbia albida & 0,00 & 0,00 & 0,00 & 0,00 & 0,00 & 0,00 & 0,19 & 0,58 & 0,00 & 0,77 & 0,09 & 0,00 & 0,00 & 0,00 & 0,09 \\
\hline Ficus dekdekena & 0,00 & 0,00 & 0,00 & 0,09 & 0,09 & 0,00 & 0,00 & 0,00 & 0,00 & 0 & 0,00 & 0,00 & 0,00 & 0,00 & 0 \\
\hline Ficus glumosa & 0,46 & 0,37 & 0,65 & 0,65 & 2,13 & 0,00 & 0,00 & 0,00 & 0,00 & 0 & 0,00 & 0,00 & 0,00 & 0,00 & 0 \\
\hline Ficus ingens & 0,00 & 0,00 & 0,55 & 0,55 & 1,10 & 0,00 & 0,00 & 0,97 & 0,00 & 0,97 & 0,00 & 0,00 & 0,00 & 0,00 & 0 \\
\hline Ficus platyphylla & 0,00 & 0,18 & 0,00 & 0,18 & 0,36 & 0,00 & 0,00 & 0,00 & 0,00 & 0 & 0,00 & 0,57 & 2,74 & 0,00 & 3,31 \\
\hline Ficus sycomorus & 0,09 & 0,55 & 0,65 & 0,46 & 1,75 & 0,00 & 0,39 & 0,39 & 0,00 & 0,78 & 0,09 & 0,00 & 0,00 & 0,00 & 0,09 \\
\hline Flueggea virosa & 0,00 & 0,00 & 0,00 & 0,00 & 0,00 & 1,56 & 7,00 & 7,98 & 3,70 & 20,24 & 0,00 & 0,00 & 0,00 & 0,28 & 0,28 \\
\hline Gardenia aqualla & 0,74 & 0,00 & 1,66 & 1,85 & 4,25 & 0,00 & 0,00 & 0,00 & 0,19 & 0,19 & 0,00 & 0,00 & 0,00 & 0,00 & 0 \\
\hline Grewia cissoides & 0,00 & 0,37 & 0,00 & 0,00 & 0,37 & 0,00 & 0,00 & 0,00 & 0,00 & 0 & 0,00 & 0,00 & 0,00 & 0,00 & 0 \\
\hline Grewia flavescens & 0,92 & 0,46 & 0,18 & 1,01 & 2,57 & 0,00 & 0,00 & 0,00 & 0,00 & 0 & 0,19 & 0,00 & 0,00 & 0,00 & 0,19 \\
\hline Grewia venusta & 0,00 & 0,00 & 0,00 & 0,00 & 0,00 & 1,17 & 0,19 & 0,00 & 1,36 & 2,72 & 1,04 & 2,08 & 2,74 & 0,57 & 6,43 \\
\hline Guiera senegalensis & 0,00 & 0,00 & 0,00 & 0,00 & 0 & 0,00 & 0,19 & 0,39 & 0,00 & 0,58 & 0,19 & 0,47 & 0,47 & 0,95 & 2,08 \\
\hline Hexalobus monopetalus & 0,18 & 1,66 & 0,46 & 1,29 & 3,59 & 0,00 & 0,19 & 0,00 & 0,19 & 0,38 & 0,00 & 1,42 & 0,76 & 0,09 & 2,27 \\
\hline Hymenocardia acida & 0,18 & 0,46 & 0,00 & 0,65 & 1,29 & 0,00 & 0,78 & 0,39 & 0,19 & 1,36 & 0,09 & 1,42 & 0,00 & 0,47 & 1,98 \\
\hline Hyparrhenia bagirmica & 0,00 & 0,00 & 0,00 & 0,00 & 0,00 & 0,00 & 0,00 & 0,19 & 0,00 & 0,19 & 0,00 & 0,09 & 0,00 & 0,00 & 0,09 \\
\hline Hyparrhenia rufa & 0,00 & 0,00 & 0,00 & 0,00 & 0,00 & 0,00 & 0,00 & 0,00 & 0,00 & 0 & 0,00 & 0,09 & 0,00 & 0,00 & 0,09 \\
\hline Hyphaene thebaica & 0,18 & 0,28 & 1,94 & 0,28 & 2,68 & 0,00 & 0,00 & 0,19 & 0,19 & 0,38 & 0,19 & 0,00 & 0,09 & 0,00 & 0,28 \\
\hline Khaya senegalensis & 0,00 & 0,28 & 0,00 & 0,00 & 0,28 & 0,00 & 0,39 & 0,39 & 0,39 & 1,17 & 0,57 & 0,00 & 0,00 & 0,28 & 0,85 \\
\hline Lannea acida & 0,00 & 0,00 & 0,00 & 0,00 & 0,00 & 0,00 & 0,19 & 0,00 & 0,19 & 0,38 & 0,00 & 0,00 & 0,28 & 0,00 & 0,28 \\
\hline Lonchocarpus laxiflorus & 0,00 & 0,00 & 0,00 & 0,00 & 0,00 & 0,00 & 0,00 & 0,00 & 0,00 & 0 & 0,00 & 0,00 & 0,00 & 0,00 & 0 \\
\hline Loudetia annua & 0,00 & 0,00 & 0,00 & 0,00 & 0,00 & 0,00 & 0,00 & 0,39 & 0,00 & 0,39 & 0,00 & 0,38 & 4,16 & 0,00 & 4,54 \\
\hline Mariscus sublimis & 0,00 & 0,00 & 0,00 & 0,00 & 0,00 & 0,00 & 0,19 & 0,00 & 0,00 & 0,19 & 0,00 & 0,00 & 0,00 & 0,00 & 0 \\
\hline Maytenus senegalensis & 0,00 & 0,00 & 0,00 & 0,00 & 0,00 & 0,00 & 0,00 & 0,00 & 0,00 & 0 & 0,00 & 0,00 & 0,00 & 0,00 & 0 \\
\hline Mimosa pigra & 0,00 & 0,28 & 0,46 & 0,09 & 0,83 & 0,00 & 0,00 & 0,58 & 0,00 & 0,58 & 0,00 & 0,00 & 0,00 & 0,00 & 0 \\
\hline Mitragyna inermis & 0,00 & 0,00 & 0,00 & 0,00 & 0,00 & 0,00 & 0,39 & 0,58 & 0,00 & 0,97 & 0,57 & 3,97 & 3,60 & 0,76 & 8,9 \\
\hline Moringa oleifera & 0,37 & 0,00 & 0,18 & 0,28 & 0,83 & 0,19 & 0,19 & 0,58 & 0,00 & 0,96 & 0,00 & 0,00 & 0,00 & 0,00 & 0 \\
\hline Oxytenanthera abyssinica & 0,00 & 0,00 & 0,00 & 0,00 & 0,00 & 0,00 & 0,00 & 0,00 & 0,19 & 0,19 & 3,12 & 0,28 & 0,38 & 4,26 & 8,04 \\
\hline Ozoroa pulcherrima & 0,00 & 0,00 & 0,00 & 0,00 & 0,00 & 0,00 & 0,00 & 0,00 & 0,00 & 0 & 0,00 & 0,00 & 0,00 & 0,00 & 0 \\
\hline Ozoroa insignis & 0,00 & 0,00 & 0,00 & 0,00 & 0,00 & 0,00 & 0,00 & 0,39 & 0,00 & 0,39 & 0,09 & 3,41 & 3,69 & 0,00 & 7,19 \\
\hline Panicum anabaptistum & 0,00 & 0,00 & 0,00 & 0,00 & 0,00 & 0,00 & 0,39 & 0,00 & 0,00 & 0,39 & 0,00 & 0,38 & 0,00 & 0,00 & 0,38 \\
\hline Parinari curatellifolia & 0,28 & 0,46 & 0,00 & 0,46 & 1,20 & 0,00 & 0,00 & 0,00 & 0,00 & 0 & 0,00 & 0,00 & 0,00 & 0,00 & 0 \\
\hline Parkia biglobosa & 1,85 & 3,42 & 2,40 & 2,31 & 9,98 & 0,00 & 0,00 & 0,00 & 0,00 & 0 & 0,00 & 0,00 & 0,00 & 0,00 & 0 \\
\hline Pennisetum atrichum & 0,00 & 0,00 & 0,00 & 0,00 & 0,00 & 0,00 & 0,00 & 0,00 & 0,00 & 0 & 0,00 & 0,19 & 0,57 & 0,76 & 1,52 \\
\hline Pericopsis laxiflora & 0,00 & 0,09 & 0,00 & 0,00 & 0,09 & 0,00 & 0,00 & 0,00 & 0,00 & 0 & 0,09 & 0,28 & 0,00 & 0,09 & 0,46 \\
\hline Piliostigma thonningii & 0,00 & 0,00 & 0,00 & 0,00 & 0,00 & 0,00 & 0,78 & 0,00 & 0,39 & 1,17 & 0,09 & 2,27 & 2,65 & 0,00 & 5,01 \\
\hline Prosopis africana & 0,00 & 0,00 & 0,00 & 0,00 & 0,00 & 0,19 & 0,78 & 0,39 & 0,58 & 1,94 & 1,70 & 2,27 & 2,37 & 1,70 & 8,04 \\
\hline
\end{tabular}




\begin{tabular}{|c|c|c|c|c|c|c|c|c|c|c|c|c|c|c|c|}
\hline \multirow{2}{*}{ Espèces } & \multicolumn{5}{|c|}{ Feeding } & \multicolumn{5}{|c|}{ Fodder } & \multicolumn{5}{|c|}{ Service } \\
\hline & $\mathbf{A}$ & B & $\mathrm{C}$ & D & $\mathbf{T}$ & $\mathbf{A}$ & $\mathbf{B}$ & $\mathbf{C}$ & D & $\mathbf{T}$ & $\mathbf{A}$ & B & $\mathbf{C}$ & D & $\mathbf{T}$ \\
\hline Pterocarpus erinaceus & 0,09 & 0,46 & 0,09 & 0,55 & 1,19 & 0,00 & 0,39 & 0,19 & 1,17 & 1,75 & 0,09 & 0,00 & 0,00 & 0,09 & 0,18 \\
\hline Rottboellia cochinchinensis & 0,00 & 0,00 & 0,00 & 0,00 & 0,00 & 0,00 & 0,39 & 0,00 & 0,00 & 0,39 & 0,00 & 0,00 & 0,00 & 0,00 & 0 \\
\hline Saba senegalensis & 0,09 & 0,00 & 0,00 & 0,00 & 0,09 & 0,00 & 0,19 & 0,00 & 0,00 & 0,19 & 0,00 & 0,00 & 0,00 & 0,00 & 0 \\
\hline Sarcocephalus latifolius & 0,09 & 0,00 & 0,09 & 0,18 & 0,36 & 0,00 & 0,00 & 0,00 & 0,00 & 0 & 0,00 & 0,47 & 0,09 & 0,00 & 0,56 \\
\hline Scadoxus multiflorus & 0,18 & 0,18 & 0,74 & 0,18 & 1,28 & 0,00 & 0,00 & 0,00 & 0,00 & 0 & 0,00 & 0,00 & 0,00 & 0,00 & 0 \\
\hline Sclerocarya birrea & 0,00 & 0,09 & 0,28 & 0,09 & 0,46 & 0,00 & 0,00 & 0,00 & 0,00 & 0 & 0,09 & 0,00 & 0,00 & 0,00 & 0,09 \\
\hline Securidaca longipedunculata & 0,00 & 0,00 & 0,00 & 0,18 & 0,18 & 0,00 & 0,00 & 0,00 & 3,70 & 3,7 & 0,19 & 0,09 & 0,00 & 0,66 & 0,94 \\
\hline Senna obtusifolia & 0,00 & 0,00 & 0,00 & 0,00 & 0 & 0,00 & 0,00 & 0,39 & 0,00 & 0,39 & 0,00 & 0,00 & 0,09 & 0,09 & 0,18 \\
\hline Sida rhombifolia & 0,00 & 0,00 & 0,00 & 0,00 & 0 & 0,00 & 0,19 & 0,00 & 0,00 & 0,19 & 0,00 & 0,00 & 0,00 & 0,19 & 0,19 \\
\hline Sterculia setigera & 0,00 & 0,00 & 0,00 & 0,00 & 0 & 0,97 & 0,19 & 0,00 & 0,00 & 1,16 & 0,00 & 0,09 & 0,28 & 0,09 & 0,46 \\
\hline Stereospermum kunthianum & 0,00 & 0,00 & 0,00 & 0,00 & 0 & 0,78 & 1,75 & 1,75 & 1,56 & 5,84 & 0,28 & 0,00 & 0,00 & 0,00 & 0,28 \\
\hline Strychnos innocua & 0,00 & 1,11 & 0,46 & 1,75 & 3,32 & 0,00 & 0,00 & 0,00 & 0,97 & 0,97 & 0,00 & 0,38 & 0,09 & 0,09 & 0,56 \\
\hline Strychnos spinosa & 0,28 & 0,09 & 0,00 & 0,92 & 1,29 & 0,00 & 0,00 & 0,00 & 0,39 & 0,39 & 0,00 & 0,00 & 0,00 & 0,00 & 0 \\
\hline Swartzia madagascariensis & 0,18 & 0,00 & 0,37 & 0,46 & 1,01 & 0,00 & 0,00 & 0,00 & 0,00 & 0 & 0,00 & 0,00 & 0,09 & 0,19 & 0,28 \\
\hline Tacca leontopetaloides & 0,18 & 0,00 & 0,18 & 0,00 & 0,36 & 0,00 & 0,00 & 0,00 & 0,00 & 0 & 0,00 & 0,00 & 0,00 & 0,19 & 0,19 \\
\hline Tamarindus indica & 1,94 & 0,83 & 1,48 & 1,11 & 5,36 & 0,39 & 0,00 & 0,39 & 0,39 & 1,17 & 0,09 & 0,00 & 0,00 & 0,00 & 0,09 \\
\hline Temnocalyx obovatus & 0,18 & 0,18 & 0,09 & 0,00 & 0,45 & 0,19 & 1,56 & 1,75 & 0,78 & 4,28 & 0,00 & 0,00 & 0,00 & 0,00 & 0 \\
\hline Tephrosia deflexa & 0,00 & 0,00 & 0,00 & 0,00 & 0 & 0,58 & 4,47 & 2,33 & 1,17 & 8,55 & 0,00 & 0,00 & 0,00 & 0,19 & 0,19 \\
\hline Terminalia laxiflora & 0,00 & 0,00 & 0,00 & 0,00 & 0 & 0,00 & 0,00 & 0,00 & 0,00 & 0 & 1,80 & 3,22 & 2,37 & 0,28 & 7,67 \\
\hline Trema orientalis & 0,00 & 0,00 & 0,00 & 0,00 & 0 & 0,00 & 0,00 & 0,00 & 0,00 & 0 & 0,76 & 0,28 & 0,09 & 0,85 & 1,98 \\
\hline Vitellaria paradoxa & 2,49 & 4,16 & 1,94 & 3,42 & 12,01 & 0,19 & 0,78 & 0,00 & 0,19 & 1,16 & 0,38 & 0,09 & 0,00 & 0,00 & 0,47 \\
\hline Vitex doniana & 0,65 & 2,31 & 1,48 & 1,11 & 5,55 & 0,39 & 1,95 & 0,58 & 0,78 & 3,7 & 0,00 & 0,00 & 0,00 & 0,00 & 0 \\
\hline Ximenia americana & 0,18 & 2,22 & 2,03 & 1,38 & 5,81 & 0,00 & 0,00 & 0,00 & 0,00 & 0 & 0,00 & 0,00 & 0,00 & 0,00 & 0 \\
\hline Ziziphus abyssinica & 1,01 & 1,20 & 3,05 & 1,38 & 6,64 & 0,00 & 0,39 & 0,39 & 0,00 & 0,78 & 0,47 & 0,00 & 0,47 & 0,19 & 1,13 \\
\hline Total & 16,28 & 27,75 & 27,58 & 28,2 & 99,81 & 11,86 & 32,42 & 28,93 & 26,62 & 99,83 & 16,69 & 29,57 & 34,39 & 19,17 & 99,82 \\
\hline
\end{tabular}

Table 3. Continue.

\begin{tabular}{|c|c|c|c|c|c|c|c|c|c|c|c|}
\hline \multirow{2}{*}{ Espèces } & \multicolumn{5}{|c|}{ Source of energy } & \multicolumn{6}{|c|}{ Pharmacopoeia } \\
\hline & $\mathbf{A}$ & $\mathbf{B}$ & $\mathbf{C}$ & D & $\mathbf{T}$ & $\mathbf{A}$ & B & $\mathbf{C}$ & D & $\mathbf{T}$ & GT \\
\hline Acacia sieberiana & 0,00 & 1,60 & 1,20 & 0,13 & 2,93 & 0,63 & 0,31 & 0,00 & 0,16 & 1,1 & 5,00 \\
\hline Afzelia africana & 0,13 & 0,13 & 0,00 & 0,67 & 0,93 & 0,00 & 0,31 & 0,00 & 0,16 & 0,47 & 10,12 \\
\hline Albizia adianthifolia & 0,00 & 0,00 & 0,00 & 0,00 & 0 & 0,00 & 0,00 & 0,00 & 0,16 & 0,16 & 0,16 \\
\hline Allophylus africanus & 0,00 & 0,00 & 0,00 & 0,00 & 0 & 0,00 & 0,00 & 0,00 & 0,31 & 0,31 & 0,40 \\
\hline Amblygonocarpus andongensis & 0,00 & 0,13 & 0,00 & 0,27 & 0,4 & 0,00 & 0,16 & 0,00 & 0,31 & 0,47 & 1,62 \\
\hline Anchomanes difformis & 0,00 & 0,00 & 0,00 & 0,00 & 0 & 0,00 & 0,63 & 1,41 & 0,00 & 2,04 & 2,04 \\
\hline Annona senegalensis & 0,00 & 0,00 & 0,00 & 0,00 & 0 & 0,00 & 0,31 & 0,00 & 0,31 & 0,62 & 4,61 \\
\hline Anogeissus leiocarpa & 4,40 & 2,40 & 5,73 & 6,53 & 19,06 & 1,41 & 1,10 & 2,04 & 1,41 & 5,96 & 42,36 \\
\hline Balanites aegyptiaca & 0,40 & 0,00 & 0,53 & 0,00 & 0,93 & 0,00 & 0,16 & 0,63 & 0,47 & 1,26 & 6,44 \\
\hline Bauhinia rufescens & 0,00 & 0,00 & 0,00 & 0,00 & 0 & 0,00 & 0,00 & 0,16 & 0,00 & 0,16 & 0,16 \\
\hline Boerhavia erecta & 0,00 & 0,00 & 0,00 & 0,00 & 0 & 0,00 & 0,00 & 0,00 & 0,00 & 0 & 0,19 \\
\hline Borassus aethiopum & 0,00 & 0,00 & 0,00 & 0,00 & 0 & 0,00 & 0,00 & 0,00 & 0,00 & 0 & 1,47 \\
\hline Bridelia ferruginea & 0,00 & 0,13 & 0,00 & 0,13 & 0,26 & 0,16 & 0,78 & 0,00 & 0,16 & 1,1 & 6,12 \\
\hline Capparis sepiaria var fischeri & 0,00 & 0,00 & 0,00 & 0,00 & 0 & 0,00 & 0,00 & 0,16 & 0,00 & 0,16 & 0,16 \\
\hline Cassia occidentalis & 0,00 & 0,00 & 0,00 & 0,00 & 0 & 0,16 & 0,31 & 0,63 & 0,16 & 1,26 & 1,26 \\
\hline Cassia sieberiana & 0,00 & 0,67 & 0,67 & 0,13 & 1,47 & 0,31 & 0,63 & 2,98 & 0,47 & 4,39 & 7,19 \\
\hline Celtis integrifolia & 0,00 & 0,13 & 0,00 & 0,00 & 0,13 & 0,00 & 0,00 & 0,16 & 0,00 & 0,16 & 1,65 \\
\hline Cissus populnea & 0,00 & 0,00 & 0,00 & 0,00 & 0 & 0,00 & 0,00 & 0,00 & 0,00 & 0 & 0,95 \\
\hline Combretum collinum & 1,47 & 2,67 & 2,67 & 4,13 & 10,94 & 0,63 & 1,10 & 0,78 & 1,88 & 4,39 & 17,43 \\
\hline Combretum glutinosum & 0,27 & 0,80 & 3,07 & 0,40 & 4,54 & 0,16 & 0,31 & 1,41 & 0,16 & 2,04 & 6,95 \\
\hline Combretum molle & 0,00 & 0,00 & 0,00 & 0,00 & 0 & 0,16 & 0,16 & 0,00 & 0,00 & 0,32 & 0,70 \\
\hline Combretum nigricans & 0,00 & 0,13 & 0,00 & 0,00 & 0,13 & 0,00 & 0,00 & 0,00 & 0,00 & 0 & 0,41 \\
\hline Commiphora pedunculata & 0,00 & 0,00 & 0,00 & 0,00 & 0 & 0,00 & 0,16 & 0,16 & 0,31 & 0,63 & 0,63 \\
\hline Cordia africana & 0,00 & 0,00 & 0,00 & 0,00 & 0 & 0,00 & 0,00 & 0,00 & 0,16 & 0,16 & 0,25 \\
\hline Crossopteryx febrifuga & 0,00 & 1,60 & 0,00 & 0,13 & 1,73 & 0,47 & 1,73 & 0,00 & 1,25 & 3,45 & 5,74 \\
\hline Cymbopogon giganteus & 0,00 & 0,00 & 0,00 & 0,00 & 0 & 0,00 & 0,00 & 0,16 & 0,47 & 0,63 & 0,81 \\
\hline Daniellia oliveri & 0,00 & 1,07 & 0,13 & 0,27 & 1,47 & 0,16 & 0,78 & 0,00 & 0,31 & 1,25 & 3,97 \\
\hline Detarium microcarpum & 3,07 & 0,40 & 0,00 & 2,40 & 5,87 & 0,63 & 0,31 & 0,78 & 0,78 & 2,5 & 20,11 \\
\hline Ectadiopsis oblongifolia & 0,00 & 0,00 & 0,00 & 0,00 & 0 & 0,00 & 0,00 & 0,00 & 0,00 & 0 & 0,77 \\
\hline Entada africana & 0,00 & 0,00 & 0,00 & 0,00 & 0 & 0,00 & 0,00 & 0,16 & 0,47 & 0,63 & 1,21 \\
\hline Eragrostis atrovirens & 0,00 & 0,00 & 0,00 & 0,00 & 0 & 0,00 & 0,00 & 0,00 & 0,00 & 0 & 0,00 \\
\hline
\end{tabular}




\begin{tabular}{|c|c|c|c|c|c|c|c|c|c|c|c|}
\hline \multirow{2}{*}{ Espèces } & \multicolumn{5}{|c|}{ Source of energy } & \multicolumn{6}{|c|}{ Pharmacopoeia } \\
\hline & $\mathbf{A}$ & B & $\mathbf{C}$ & D & $\mathbf{T}$ & $\mathbf{A}$ & B & $\mathrm{C}$ & D & $\mathbf{T}$ & GT \\
\hline Eragrostis ciliaris & 0,00 & 0,00 & 0,00 & 0,00 & 0 & 0,00 & 0,00 & 0,16 & 0,00 & 0,16 & 0,63 \\
\hline Euphorbia kamerunica & 0,00 & 0,00 & 0,00 & 0,00 & 0 & 0,00 & 0,00 & 0,00 & 0,00 & 0 & 0,27 \\
\hline Faidherbia albida & 0,13 & 0,00 & 0,13 & 0,00 & 0,26 & 0,31 & 0,16 & 0,47 & 0,16 & 1,1 & 2,22 \\
\hline Ficus dekdekena & 0,00 & 0,00 & 0,00 & 0,00 & 0 & 0,00 & 0,00 & 0,00 & 0,00 & 0 & 0,09 \\
\hline Ficus glumosa & 0,00 & 0,27 & 0,13 & 0,00 & 0,4 & 0,16 & 0,00 & 0,16 & 0,00 & 0,32 & 2,85 \\
\hline Ficus ingens & 0,00 & 0,00 & 0,27 & 0,00 & 0,27 & 0,00 & 0,00 & 0,63 & 0,16 & 0,79 & 3,13 \\
\hline Ficus platyphylla & 0,00 & 0,13 & 0,00 & 0,00 & 0,13 & 0,00 & 0,31 & 0,16 & 0,31 & 0,78 & 4,58 \\
\hline Ficus sycomorus & 0,00 & 0,00 & 0,00 & 0,00 & 0 & 0,16 & 0,47 & 0,94 & 0,16 & 1,73 & 4,35 \\
\hline Flueggea virosa & 0,00 & 0,00 & 0,00 & 0,00 & 0 & 0,00 & 0,31 & 0,47 & 0,00 & 0,78 & 21,3 \\
\hline Gardenia aqualla & 0,00 & 0,00 & 0,13 & 0,00 & 0,13 & 0,00 & 0,00 & 0,31 & 0,16 & 0,47 & 5,04 \\
\hline Grewia cissoides & 0,00 & 0,00 & 0,00 & 0,00 & 0 & 0,00 & 0,00 & 0,00 & 0,00 & 0 & 0,37 \\
\hline Grewia flavescens & 0,00 & 0,00 & 0,00 & 0,00 & 0 & 0,00 & 0,00 & 0,00 & 0,31 & 0,31 & 3,07 \\
\hline Grewia venusta & 0,27 & 0,00 & 0,00 & 0,00 & 0,27 & 0,00 & 0,00 & 0,00 & 0,00 & 0 & 9,42 \\
\hline Guiera senegalensis & 0,27 & 0,00 & 0,93 & 0,13 & 1,33 & 0,47 & 0,63 & 0,94 & 0,63 & 2,67 & 6,66 \\
\hline Hexalobus monopetalus & 0,00 & 0,93 & 0,13 & 0,27 & 1,33 & 0,00 & 0,31 & 0,00 & 0,31 & 0,62 & 8,19 \\
\hline Hymenocardia acida & 0,67 & 1,87 & 0,00 & 2,40 & 4,94 & 1,72 & 1,25 & 0,63 & 2,66 & 6,26 & 15,83 \\
\hline Hyparrhenia bagirmica & 0,00 & 0,00 & 0,00 & 0,00 & 0 & 0,00 & 0,00 & 0,00 & 0,00 & 0 & 0,28 \\
\hline Hyparrhenia rufa & 0,00 & 0,00 & 0,00 & 0,00 & 0 & 0,00 & 0,00 & 0,00 & 0,00 & 0 & 0,09 \\
\hline Hyphaene thebaica & 0,27 & 0,00 & 0,13 & 0,00 & 0,4 & 0,00 & 0,16 & 0,00 & 0,47 & 0,63 & 4,37 \\
\hline Khaya senegalensis & 0,13 & 0,53 & 0,67 & 0,27 & 1,6 & 0,63 & 0,00 & 0,31 & 0,63 & 1,57 & 5,47 \\
\hline Lannea acida & 0,00 & 0,00 & 0,00 & 0,00 & 0 & 0,00 & 0,00 & 0,00 & 0,31 & 0,31 & 0,97 \\
\hline Lonchocarpus laxiflorus & 0,00 & 0,00 & 0,00 & 0,00 & 0 & 0,00 & 0,00 & 0,00 & 0,00 & 0 & 0,00 \\
\hline Loudetia annua & 0,00 & 0,00 & 0,00 & 0,00 & 0 & 0,00 & 0,00 & 0,00 & 0,00 & 0 & 4,93 \\
\hline Mariscus sublimis & 0,00 & 0,00 & 0,00 & 0,00 & 0 & 0,00 & 0,16 & 0,00 & 0,00 & 0,16 & 0,35 \\
\hline Maytenus senegalensis & 0,00 & 0,00 & 0,00 & 0,00 & 0 & 0,00 & 0,16 & 0,00 & 0,00 & 0,16 & 0,16 \\
\hline Mimosa pigra & 0,00 & 0,00 & 0,00 & 0,00 & 0 & 0,00 & 0,94 & 0,00 & 0,00 & 0,94 & 2,35 \\
\hline Mitragyna inermis & 0,27 & 2,00 & 2,00 & 0,27 & 4,54 & 0,47 & 0,78 & 0,94 & 0,47 & 2,66 & 17,07 \\
\hline Moringa oleifera & 0,00 & 0,00 & 0,00 & 0,00 & 0 & 0,47 & 0,63 & 0,16 & 0,16 & 1,42 & 3,21 \\
\hline Oxytenanthera abyssinica & 0,13 & 0,00 & 0,00 & 0,00 & 0,13 & 0,00 & 0,00 & 0,00 & 0,00 & 0 & 8,36 \\
\hline Ozoroa pulcherrima & 0,00 & 0,00 & 0,00 & 0,00 & 0 & 0,16 & 0,00 & 0,00 & 0,00 & 0,16 & 0,16 \\
\hline Ozoroa insignis & 0,00 & 0,00 & 0,00 & 0,00 & 0 & 0,00 & 0,00 & 0,00 & 0,00 & 0 & 7,58 \\
\hline Panicum anabaptistum & 0,00 & 0,00 & 0,00 & 0,00 & 0 & 0,00 & 0,00 & 0,16 & 0,00 & 0,16 & 0,93 \\
\hline Parinari curatellifolia & 0,13 & 0,00 & 0,00 & 0,00 & 0,13 & 0,16 & 0,00 & 0,00 & 0,00 & 0,16 & 1,49 \\
\hline Parkia biglobosa & 0,00 & 0,80 & 0,00 & 0,27 & 1,07 & 0,31 & 0,47 & 0,78 & 0,31 & 1,87 & 12,92 \\
\hline Pennisetum atrichum & 0,00 & 0,00 & 0,00 & 0,13 & 0,13 & 0,00 & 0,16 & 0,00 & 0,00 & 0,16 & 1,81 \\
\hline Pericopsis laxiflora & 0,00 & 0,00 & 0,00 & 0,27 & 0,27 & 0,00 & 0,31 & 0,00 & 0,16 & 0,47 & 1,29 \\
\hline Piliostigma thonningii & 0,67 & 1,60 & 1,07 & 0,00 & 3,34 & 0,78 & 1,73 & 0,94 & 1,88 & 5,33 & 14,85 \\
\hline Prosopis africana & 0,93 & 1,47 & 1,73 & 1,73 & 5,86 & 0,47 & 0,47 & 0,63 & 1,25 & 2,82 & 18,66 \\
\hline Pterocarpus erinaceus & 0,00 & 0,00 & 0,00 & 1,20 & 1,2 & 0,00 & 0,00 & 0,00 & 0,63 & 0,63 & 4,95 \\
\hline Rottboellia cochinchinensis & 0,13 & 0,00 & 0,00 & 0,00 & 0,13 & 0,00 & 0,00 & 0,00 & 0,00 & 0 & 0,52 \\
\hline Saba senegalensis & 0,00 & 0,00 & 0,00 & 0,00 & 0 & 0,00 & 0,00 & 0,00 & 0,00 & 0 & 0,28 \\
\hline Sarcocephalus latifolius & 0,00 & 0,00 & 0,00 & 0,00 & 0 & 0,16 & 1,57 & 1,88 & 0,78 & 4,39 & 5,31 \\
\hline Scadoxus multiflorus & 0,00 & 0,00 & 0,00 & 0,00 & 0 & 0,00 & 0,00 & 0,00 & 0,00 & 0 & 1,28 \\
\hline Sclerocarya birrea & 0,00 & 0,00 & 0,00 & 0,00 & 0 & 0,00 & 0,47 & 0,31 & 0,31 & 1,09 & 1,64 \\
\hline Securidaca longipedunculata & 0,00 & 0,00 & 0,00 & 0,00 & 0 & 0,16 & 0,47 & 0,63 & 0,47 & 1,73 & 6,55 \\
\hline Senna obtusifolia & 0,00 & 0,00 & 0,00 & 0,00 & 0 & 0,00 & 0,00 & 0,16 & 0,00 & 0,16 & 0,73 \\
\hline Sida rhombifolia & 0,00 & 0,00 & 0,00 & 0,00 & 0 & 0,00 & 0,00 & 0,00 & 0,00 & 0 & 0,38 \\
\hline Sterculia setigera & 0,00 & 0,00 & 0,00 & 0,00 & 0 & 0,47 & 0,00 & 0,16 & 0,00 & 0,63 & 2,25 \\
\hline Stereospermum kunthianum & 0,27 & 0,13 & 0,00 & 0,53 & 0,93 & 0,47 & 0,16 & 0,16 & 0,00 & 0,79 & 7,84 \\
\hline Strychnos innocua & 0,00 & 0,13 & 0,00 & 0,00 & 0,13 & 0,00 & 0,00 & 0,00 & 0,16 & 0,16 & 5,14 \\
\hline Strychnos spinosa & 0,00 & 0,00 & 0,00 & 0,13 & 0,13 & 0,00 & 0,16 & 0,00 & 0,16 & 0,32 & 2,13 \\
\hline Swartzia madagascariensis & 0,00 & 0,00 & 0,00 & 0,27 & 0,27 & 0,00 & 0,31 & 0,16 & 0,00 & 0,47 & 2,03 \\
\hline Tacca leontopetaloides & 0,00 & 0,00 & 0,00 & 0,00 & 0 & 0,00 & 0,16 & 0,00 & 0,16 & 0,32 & 0,87 \\
\hline Tamarindus indica & 0,00 & 0,00 & 0,27 & 0,00 & 0,27 & 0,94 & 0,31 & 0,47 & 0,47 & 2,19 & 9,08 \\
\hline Temnocalyx obovatus & 0,00 & 0,00 & 0,00 & 0,00 & 0 & 0,16 & 0,00 & 0,00 & 0,16 & 0,32 & 5,05 \\
\hline Tephrosia deflexa & 0,00 & 0,00 & 0,00 & 0,00 & 0 & 0,00 & 0,31 & 0,00 & 0,00 & 0,31 & 9,05 \\
\hline Terminalia laxiflora & 2,27 & 4,00 & 3,87 & 2,27 & 12,41 & 1,10 & 2,66 & 1,41 & 1,72 & 6,89 & 26,97 \\
\hline Trema orientalis & 0,13 & 0,00 & 0,13 & 0,00 & 0,26 & 0,00 & 0,00 & 0,00 & 0,00 & 0 & 2,24 \\
\hline Vitellaria paradoxa & 1,07 & 1,87 & 0,00 & 0,93 & 3,87 & 0,31 & 1,25 & 0,16 & 0,47 & 2,19 & 19,70 \\
\hline Vitex doniana & 0,00 & 0,27 & 0,13 & 0,00 & 0,4 & 1,10 & 0,00 & 0,00 & 1,25 & 2,35 & 12,00 \\
\hline Ximenia americana & 0,00 & 0,00 & 0,13 & 0,00 & 0,13 & 0,00 & 1,25 & 0,47 & 0,00 & 1,72 & 7,66 \\
\hline Ziziphus abyssinica & 0,53 & 0,00 & 0,93 & 0,00 & 1,46 & 0,16 & 0,78 & 0,31 & 0,16 & 1,41 & 11,42 \\
\hline Total & 18,14 & 27,99 & 27,05 & 26,79 & 99,97 & 16,5 & 28,99 & 26,69 & 27,9 & 100,08 & 499,51 \\
\hline
\end{tabular}

A: Niellim, B: Balimba, C: Kokaga, D: Djoli, T: Total, GT: GrandTotal. 


\section{Discussion}

\subsection{Characteristics of the Sample}

This study highlighted the evolution of the MNP as perceived by the riparians populations based on a survey conducted among a sample of people of diverse origins in 4 riparians townships. The sample of respondents was largely made up of men, about three times more (74\%) than women $(26 \%)$. This can be explained by the availability of men to provide information compared to women who remain in the marital home. This is because tradition dictates that the man is not only the head of the household but also the spokesperson for the household. In addition, there is a limit to the mixing of men and women, which is also part of the local culture. This result confirms those of [16, 17-18] who all showed that men predominate over women in their sample of respondents and this proportion is linked to local tradition in the Far North of Cameroon and the Chari in Chad. The majority of the respondents have an average age between 25 and 40 years (39\% of the answers), followed by those between 40 and 55 years $(36.50 \%)$. People over 55 years old are less numerous in the samples. It is estimated at $24.5 \%$ on average. This result corroborates that of [19] who worked on "Impact of wood energy exploitation on wood resources in Gadala and its periphery" and that of [20] on "Anthropization and spatio-temporal dynamics of forest landscapes of the Ngaoundéré cliff (Adamaoua-Cameroon)". both showed that adults [25-50] are the most represented during their different investigations, but in disagreement with that of [21] who showed in his study entitled "Impact of deforestation on vegetation dynamics in the Sudano-Sahelian zone of Cameroon" that it is rather young people ( $\leq 25$ years) who are much more represented. This difference could be related to the initial age set by the different authors, the different ethnic groups surveyed and their traditional weight. The high rate of married people in the four (04) townships is due to the age that we had set at a minimum of 25 years for our survey. At this age, both boys and girls are already married; this is also due to the culture of the area and early marriages of young people. This result corroborates that of [18] who found $95.8 \%$ married, $1.9 \%$ widowed, $0.6 \%$ single and $1.7 \%$ divorced in their sample of respondents. The high primary school rate (52\%) in Balimba and Djoli can be explained by the fact that parents send their children to school up to primary school level, but from secondary school onwards, parents can no longer afford the school fees, because not only are the colleges far from the villages, but the school fees are high for the villagers. Parents keep their children busy with field work, especially harvesting. The latter usually ends in mid-January and school begins in October of each year. On the other hand, the average rate in Niellim (28\%) and Kokaga (34\%) townships can be explained by the fact that parents prefer to send their children to Koranic school rather than to French school, because the two townships have a majority of Muslims, or it may be due to the work in the fields that keeps them busy and prevents them from attending school. The illiteracy rate (37\%) for all four townships, especially in Kokaga (54\%) and Niellim (50\%), shows that the majority of respondents have not had access to school. This may be due to poverty, as was often mentioned by respondents during the interviews. In general, in rural areas, very few people reach secondary or even higher education. This is due to the fact that most people are not only confronted with financial problems, but also with the distance to school and the lack of teachers. In this context, these populations declare that they find their accounts much more in activities such as agropastoral and the exploitation of forest resources. This result is similar to those of [18] and [22] who showed that it is the illiterate who have a high rate of schooling compared to other levels of study.

\subsection{Anthropogenic Pressure on Park Vegetation}

The socio-economic activities of MNP riparians result in significant pressures on the area's woody resources and their habitat [23]. The increasing demand for agro-pastoral land and the inadequacy of management practices are all factors that considerably reduce the presence of plants and their essential resources in the Sahel [24], which includes Chad. These different anthropogenic pressures on the stands induce a modification of their structure and accentuate their degradation. Grazing is much more common in and around the park, followed by poaching and bushfires. All townships are affected by illegal resource harvesting. These are due to the increased demand in urban areas. In Sarh especially, which is a large town, the need for firewood, including charcoal, covers at most three quarters of consumption. In contrast, in rural areas where there is no electricity and where petroleum and gasoil are not widely available to fuel lamps in the rainy season, more than $1.5 \mathrm{~m}^{3}$ of wood are consumed per capita per year [25]. This harvesting takes place mainly in the dry season. In fact, there are many access roads around and within the MNP that facilitate the penetration and circulation of populations. This ease of access is also linked to the existence of multiple trails developed by the forestry administration and by poachers and fishermen. It is true that bush fires destroy vegetation, but many scientists admit that they do not destroy the savannahs. On the contrary, they are the main factors in maintaining savannahs. The total suppression of fires or the practice of early fires will thus make the savannahs evolve towards more closed environments and would modify the physiognomy and composition of the vegetation, as well as the balance of the ecosystem as a whole [26, 27]. Bushfires are a key element in the dynamics of savannah landscapes in Sudanian regions [26, 27] and are considered, depending on the ecological zones concerned, either as a scourge to be combated or as a genuine management tool, the reasoned use of which makes it possible to maintain or sustain certain types of landscapes and, consequently, some of the populations that are dependent on them. For the wood resource, the removal devices are not always feasible with regeneration and growth. On average, these are topping (23.54\%), debarking (14.02\%), 
wood gathering (23.01\%), pruning (16.93\%), and cutting of wood at ground level $(22.48 \%)$. These pressures have environmental, economic and social consequences. For economic reasons, even fruit species such as Vitelleria paradoxa and Tamarindus indica are now sacrificed [28]. Another disturbing fact is that in order to feed their livestock, herders cut down or uproot outright some species such as Afzelia africana, Anogeissus leiocarpus, Stereospermum kunthianum, etc (Figures 6 and 7). Indeed, the increasing impoverishment of rural populations, which represent $80 \%$ of the Chadian population (rural poverty index: $70.3 \%$ ), leads them to increase pressure on the natural resources of the MNP, the only easily accessible sources that can be consumed or directly generate income [29]. In this sense, without alternative sources of income generation or fuel or other service materials, the pressure will further increase on the phytodiversity which will be difficult to manage sustainably [29]. These anthropic pressures are often also due to the State's lack of understanding of the real needs of the populations living near protected areas, which translates into a lack or inadequacy of support measures and economic incentives for the populations to improve their living conditions [29]. Nowadays, we are witnessing an increasingly marked sedentarization of herders, coming from the north and center of Chad. In Mali, in the Baoule National Park, human settlements for grazing and agriculture are such that a partial declassification has been considered. This sedentarization is a risk for the torpid Manda National Park [25]. New socio-economic activities such as tree cutting with electric chainsaws, trade in fuelwood, organized poaching by well-armed vehicular groups, and illegal fishing increase this pressure on MNP's natural resources. The remote causes of biodiversity degradation in MNP are climatic hazards, particularly poor rainfall distribution (irregularities, shortages, early cessation of the rainy season) [30].

\subsection{Dynamics of the Vegetation of MNP use of Between 2009-2019}

Overall, according to the respondents, the vegetation area of the park and its density are decreasing (46.25\%) between 2009 and 2019. This regression is due to deforestation, the rate of which is $2.5 \%$ ha/year in Chad [31]. The MODIS VCF sensor detected that tree cover in Chad underwent a reduction of over $20 \%$ between 2000 and 2010 [31]. The increase $(36.03 \%)$ in the park's vegetation area and density may be due to the Law prohibiting the entry and cutting of timber in this park or to the good supervision of forestry agents (Eco guards). As for its stagnation (17.71\%), we can say that, despite its prohibition, recalcitrant local residents violate the law by collecting LFPs and NTFPs, thus preventing its progression. These results are similar to those of [32] who found in the Burkinabe Sahel a low proportion (9\%) of respondents who mentioned an improvement in vegetation cover over the last 20 years. According to [22], less than $2 \%$ of the respondents mentioned an increase in the area of vegetation cover in the Burkinabe Sahel over the past 30 years, and almost all of the respondents (92\%) noted a regression in the area of vegetation cover. This percentage attests to the fact that local communities share the same opinion regarding the degradation of their environment's plant resources. This trend of decreasing vegetation cover in the Sahel is explained by the adverse effects of climatic hazards, aggravated by the intense anthropic activities of local populations [22].

\subsection{The Main Resources Harvested in Manda National Park}

Of all the four townships, the species most valued for their various uses are Anogeissus leiocarpus (42.36\%), Terminalia laxiflora (26.97\%), Flueggea virosa (21.3\%), Detarium microcarpum $(40.11 \%)$, and Vitellaria paradoxa $(19.96 \%)$, but also moderately valued Parkia biglobosa (9.98\%). The main product of the shea tree (Vitellaria paradoxa) is its butter, which is an important source of oil for culinary preparations, in particular "Tan-koule", a sauce that is in great demand in both urban and rural areas. The traditional extraction of shea butter is a female activity. This species also provides the population with the pulp of its fruits, which is highly appreciated and consumed. The powdered leaves of Parkia biglobosa are well consumed; its flowers are sucked by children; the pulps of its fruits are used to make sweet drinks. These pulps are also energetic when made into pasta and consumed alone or in combination with millet flour [33]. Its seeds, especially when fermented, are a popular condiment in sauces and substitute flavors (Maggie). In general, the importance of plant species is explained by the quality and availability of their organs harvested by local populations. For Detarium microcarpum, not only are its fruit pulps, rich in vitamin C, edible and locally marketed [34]. All of these species are found in their preferred area in the four townships. In addition, in the face of recurrent instability in agricultural production, rural populations, especially women, are developing survival strategies, one of the most common of which is the use of plant products from agroforestry parks, such as fruits, flowers, seeds, leaves, bark and roots, as an alternative source of subsistence [35, 36]. However, the number of food species (48) in the study area is similar to that recorded (49 species) by Guimbo et al. [37] in the peripheral areas of Niger's Park W, but higher than that recorded ( 24 species) by Laminou et al. [23] in the Guidan Roumdji locality in Niger. This difference is probably related to the study area, which constitutes a large area characterized by a variety of ecosystems and a diversity of plant species [38, 39, 23]. Nevertheless, these species have good nutritional value and are highly valued by the populations [40, 23]. The leaves of Flueggea virosa (20.24\%), Tephrosia deflaxa $(8.55 \%)$ and Afzelia africana (8.17\%) are highly palatable to the livestock of the riparian populations of MNP because of their nutritional value. The twigs and flowers of these species are sometimes eaten by livestock. The great interest of livestock in consuming certain species of fodder plants at the expense of others stems from their instinctive ability to choose those that are beneficial to them and abandon those that seem relatively less profitable, even toxic [17]. Exploitation patterns often threaten most species, especially when the foliage is beyond 
the reach of animals. In this case, shepherds do not hesitate to mutilate or cut down a tree (Figure 6). The importance of woody plants as fodder is due to the fact that they provide proteins, minerals and vitamins that are essential to the dietary balance of livestock, and thus to the life of the herds during the dry seasons, and to their survival during abnormal drought [41, 24]. Afzelia africana leaves stimulate milk production in cows [24]. This species has a response rate of $8.17 \%$ in our study area and highly solicited by herders (Figure 6). Households around the park have recourse to wood, taken from inside or outside the park, to meet their needs for materials. The high consideration of certain species to the detriment of others is due to the interest of the local populations in meeting their most important needs. This is the case for Anogeissus leiocarpus (13.44\%), Mitragyna inermis (8.90\%), Oxytenanthere abyssinica $(8.04 \%)$ and Prosopis africana $(8.04 \%)$, which are much in demand for their water resistance. In the rural, peri-urban and urban areas, the demand for wood and straw for the construction of houses, sheds, etc., is very high. For this reason, some people make long trips to look for straw to weave the "Seko", a kind of local mat (Figures 8 and 9). In addition, for a wood to be used in this logic, the respondents mention their resistance to climatic hazards and pests. In addition, the shape of the wood, i.e., the more or less rectilinear appearance in order to form the scaffolding of the roofs or even the presumed shed in the case of construction. This result corroborates that of [17] on the fact that Anogeissus leiocarpus occupies a primordial place among the species exploited and used in various fields such as firewood, construction, shade, etc (Figures 10 and 11). The town of Sarh has a great influence on the functioning of the fuel energy source, as $98 \%$ of households in the Moyen-Chari region use wood fuels [28]. The cause of the exploitation of the most prized species emanates from the quality of their wood and their availability. The wood of these species burns slowly during use as firewood or charcoal used in cooking. Some species are increasingly rare compared to others. Eventually, people such as housekeepers, blacksmiths, butchers, brick makers, etc., are forced to use the available species. This is why Anogeissus leiocarpus (19.06\%) is very much in demand for heating purposes, given its reputation for slow burning and its availability. This result is similar to that of [28] who cited Anogeissus leiocarpus as one of the most used species in the Minawao locality, in terms of heating given its availability and reputation to burn slowly. [19] also reported in his studies that Anogeissus leiocarpus is the species used par excellence in the field of heating in the Far North of Cameroon. In general, Terminalia laxiflora (6.89\%), Hymenocardia acida (6.26\%) and Anogeissus leiocarpus (5.96\%) are exploited in traditional pharmacopoeia according to the virtues that local populations can draw from them and their knowledge. In the study area, 74 woody species have been identified as being used in traditional pharmacopoeia for a wide range of therapeutic actions. However, this number is still higher than the number (40 species) of [32], found in the Kédougou area of Senegal and the number (38 species) of [17] found in Minawao in the Mayo Tsanaga Department, Far North, Cameroon. This could be explained by the difference in ecological zone and the various socio-cultural traits of the population. However, this result corroborates those of [42] who first mentioned Cassia sieberiana (or Casse de Siéber in French and Koumbrsaka in Mooré), as the species providing important remedies and that of [21] who showed that the species cited in this context are widely used in the Sahelian zone.

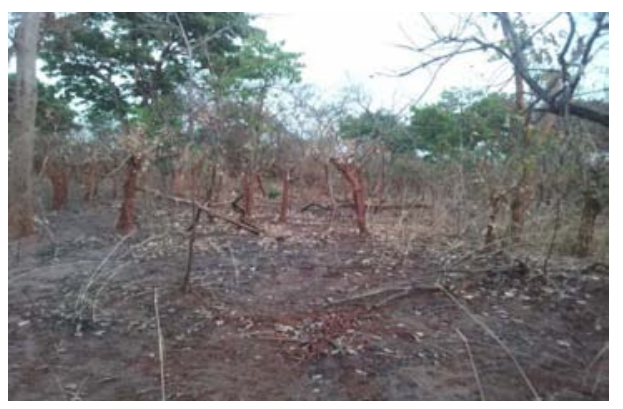

Figure 6. Pollarded sections of several individuals of Afzelia africana (Photo: waya, 2020).

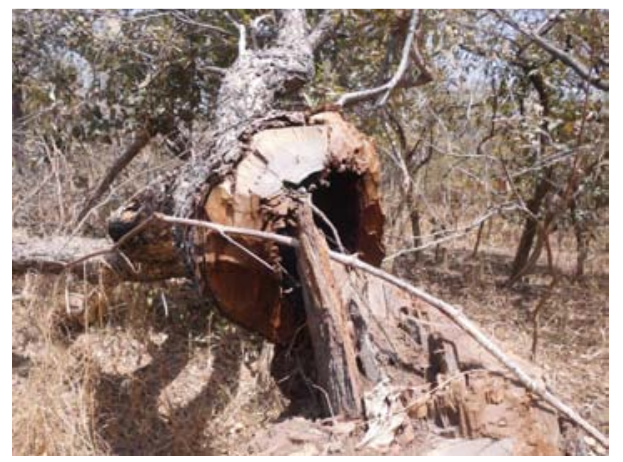

Figure 7. Flush cut of Stereospermum kunthianum (Photo: waya, 2020).

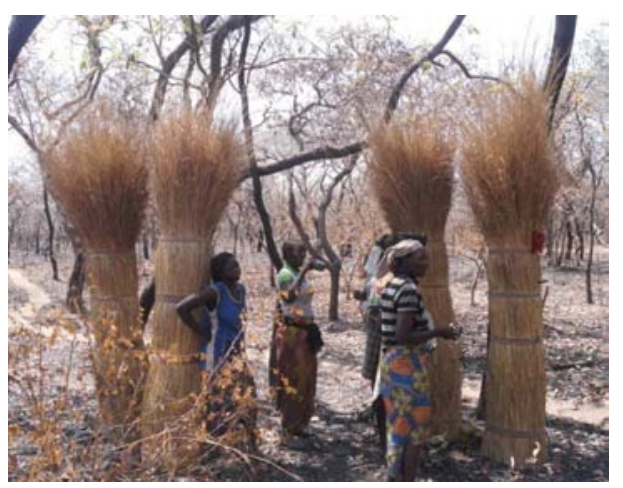

Figure 8. Women with straw serving the roof of the house (Photo: waya, 2020).

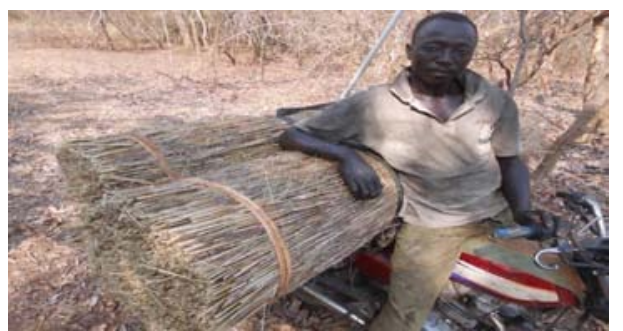

Figure 9. Straw for the "Seko" as a roof or fence for a house (Photo: waya, 2020). 


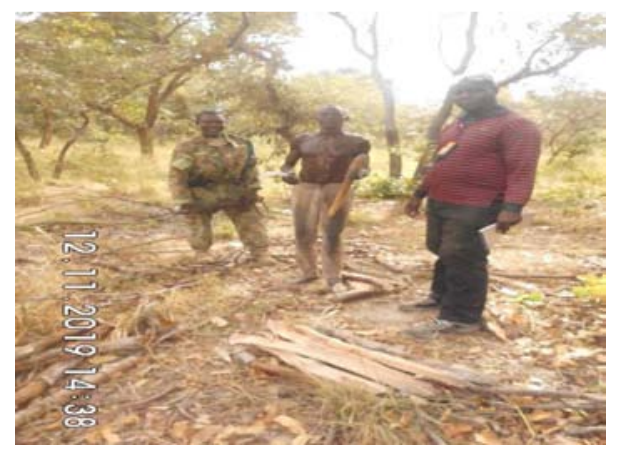

Figure 10. Man cutting firewood (photo: Waya, 2019).

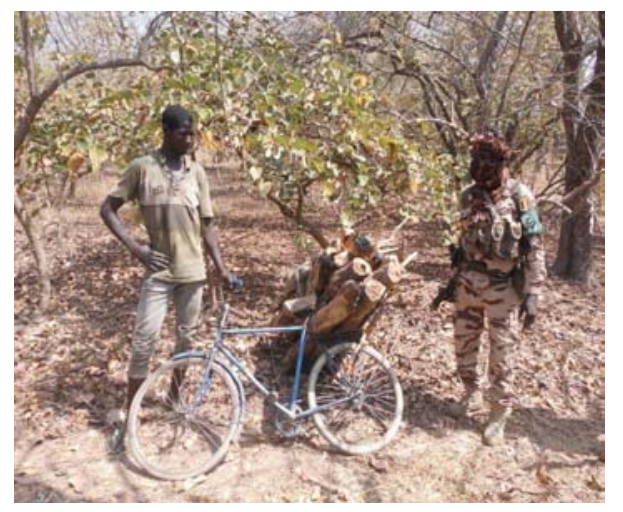

Figure 11. Man carrying firewood (Photo: Waya, 2019).

\subsection{Proposals for Sustainable Management of the MNP}

Concerning the evolution of the vegetation stand over the last ten (10) years (2009 to 2019), the MNP is in regression. This regression is confirmed by the respondents, forest managers or non-forest residents. Most of the people interviewed recognize the richness of the park's biological resources, but mention several problems that could hinder sustainable management, especially those related to the application of the management plan. This survey, conducted using a participatory approach, showed that the MNP has undergone changes in its flora and fauna. The local actors better perceive this dynamic, and even the importance of the park, by indicating the multiple resources available or the rich biodiversity. Also, the evocation of the reality of anthropic pressures highlights the inadequacies in management. Among the main causes, overgrazing and poaching are cited by almost all the actors interviewed, followed by abusive logging and, finally, illegal fishing, poor management, bush fires and the harvesting of non-timber forest products (NTFPs). A wide range of solutions were proposed by the sampled population to restore this park. Here are some of the proposals: (1) Involvement of the riparian population in the management of the MNP, through sensitization and recruitment of young people as volunteers; (2) granting of credits to the population especially women for trade in order to orient them towards other activities not related to the park; (3) application of the Law relating to the MNP in all its rigor; (4) subsidizing and/or popularizing construction materials, butane gas and agricultural products; (5) increasing the number of forestry agents and training them in new technologies so that they can properly monitor and control the park; (6) reforesting bare spaces in the MNP by introducing plant and animal species that have disappeared and/or are in danger of extinction. This principle is well understood by the local populations when a number of solutions are proposed to them for the sustainability of the park's ecosystem services. It is also important to take into account the cultural aspirations of local populations. According to [43], the cultural and spiritual values of spaces and resources cannot be separated from either planning strategies or the way of life of the local population. With this in mind, it would be beneficial to focus on sensitizing township customary and religious leaders to act as relays for raising awareness among local populations. The populations would listen to the words of a chief or a village elder more than any forestry service agent. In fact, in these localities dominated by hunters, the chief himself, who is the custodian of tradition, must call on the population to abandon poaching so that they do not feel marginalized from their culture. It is also necessary to reassure this population by saving their tradition. It is also necessary, in order to ensure the integrity of the boundaries of the protected areas, to lead the local populations towards a modernization of agriculture in order to increase yields and avoid their encroachment. This must be done through the granting of agricultural means, but also through training and regular and rigorous monitoring. Developments involving farmers would make it possible to reconcile, in particular, the production of firewood by pruning, a scarce resource, and the sustainability of tree parks $[44,16]$. [45] proposes ways to reduce pressure on wood and fodder resources by improving the energy efficiency of fireplaces, using alternative energy, reducing the number of herds and controlling grazing and fire management techniques. In Oti-Ke'ran National Park in North Togo, the sale of dead wood collected and the allocation of income to local populations are proposed to managers to facilitate participatory management $[46,16]$. There is a need to strengthen forestry agents in sufficient numbers. In Chad, there is one surveillance officer for every $507 \mathrm{~km}^{2}$, whereas the norm is one for every $25 \mathrm{~km}^{2}$ [29]. In addition, they are poorly treated in terms of salary despite the magnitude of the task entrusted to them, so that they generally have a disadvantaged social situation. This situation does not allow them to fight effectively against the bad practices of the populations, notably poaching, abusive wood cutting, etc. The same conclusion was reached in the pastoral enclave of Dadaria in Niger [22]. The weakness in the application of texts on natural resource governance has been cited as a factor favoring the accelerated degradation of these natural resources.

\section{Conclusion}

This study conducted in four townships bordering the MNP has shown that local populations have a good level of knowledge of the limit and evolution of the plant cover, as well as the causes of the degradation of this plant resource. 
The most commonly used forest resources in MNP are those used in the traditional pharmacopoeia (100.08\%). Their use as a food source is more important in Djoli (28.2\%), Kokaga (27.58\%) and Balimba (27.75\%) townships compared to Niellim (16.28\%). Fodder use is more prevalent in Balimba Township (32.42\%). On the other hand, in Kokaga Township, the use of construction materials is the highest (34.39\%). The township of Balimba exploits forest resources more as a source of fuel for energy $(29.99 \%)$ and in traditional pharmacopoeia $(28.99 \%)$. The plant species most commonly harvested in the MNP for various uses are: Anogeissus leiocarpus (42.36\%), Terminalia laxiflora (26.97\%), Flueggea virosa (21.3\%), Detarium microcarpum (20.11\%) and Vitellaria paradoxa $(19.70 \%)$. On the other hand, the most important direct determinant of vegetation cover loss is bush fires, logging and hunting. Popular perceptions produce results comparable to those of scientific studies on floristic inventories and remote sensing. Hence the importance of involving local populations in park management studies.

\section{References}

[1] Ballah S. R., 2008. Le Parc national de Manda: dynamique des ressources naturelles et cogestion, mémoire de DEA, Université de Yaoundé 1, Cameroun, 83p.

[2] UICN / WCMC, 1994. Guidelines for Protected Area Management Categories. Gland et Cambridge: UICN.

[3] Amsallem I., Kone D. P., Wilkie M. L. and Ngandji M., 2004. Gestion forestière en Afrique centrale: à la recherche de l'excellence. Bois et forêts des tropiques, 281 (3): 5-18.

[4] Badji M., Sanogo D. and Akpo L. E., 2014. Dynamique de la végétation ligneuse des espaces sylvo-pastoraux villageois mis en défens dans le Sud du Bassin arachidier au Sénégal. Bois et Forêts des Tropiques, 319 (1): 43-52.

[5] Thomassey J-P., 1991. La situation des ressources naturelles du Tchad. Bois et Forêts des Tropiques, 228: 49-62.

[6] UICN/PACO 2008. Evaluation de l'efficacité de la gestion des aires protégées: aires protégées du Tchad. 56p.

[7] Sègun O. G. H. A. 2016. Perception locale de la dynamique du couvert végétal des terroirs riverains de la réserve de biosphère du w Benin. Cas de la zone de Karimama. lère promotion Mémoire présenté en vue de l'obtention du diplôme de Licence Professionnelle en Aménagement et Gestion des Aires protégées. Université de Parakou, Centre universitaire de Kandi, Ecole Nationale Supérieure d'Aménagement et de Gestion des Aires Protégées, (ENSAGAP), Département d'Économie et de Socio-Anthropologie des Aires Protégées, $66 \mathrm{p}$.

[8] Mertens, Orekan B., Eds V., 2019. Actes de la Conférence «des images satellites pour la gestion durable des territoires en Afrique», Actes de la Conférence OSFACO, Cotonou, Bénin, $547 \mathrm{p}$.

[9] PAPNM, 2010. Plan d'aménagement du Parc National de Manda 2011-2021. 175p.

[10] Ngarnougber C., Ibrahima A., Saradoum G. and Ngaryo F. T,
2016. Caractérisation des peuplements et la phytomasse du parc national de Manda dans la région du Moyen-Chari, Tchad. Afrique SCIENCE, 12 (6): 1-13.

[11] ASECNA, 2018. Agence pour la Sécurité Aérienne en Afrique et à Madagascar, centre météorologique de sarh.

[12] Pias J., 1964. Les sols du Tchad. VIIIème Congrès International de la Science du Sol, Bucarest-Roumanie. Comptes rendus, (V): 145-151.

[13] Tchago B., 1999. Les systèmes de gestion participative rationnels avec une exploitation et une gestion optimale des ressources par l'atténuation des pressions qui existent dans et autour du Parc National de Manda. Rapport de consultation, Tchad, $108 \mathrm{p}$.

[14] Pias J., 1970. La végétation du Tchad, ses rapports avec les sols et variations paléobotaniques au quaternaire. Contribution à la connaissance du bassin Tchadien. Travaux et documents de l'0. R. S. T. O. M., n 6, 49 p.

[15] Saradoum. G., 2012. Étude phytosociologique et diagnostic faunique du Parc National de Manda au Tchad; éléments pour un aménagement. Thèse pour l'obtention du grade de Docteur en Biologie, Physiologie et Pathologie et Végétale. UCAD, DAKAR, 183p.

[16] Sandjong S. R. C., Ntoupka M., Ibrahima A. and Vroumsia T., 2013. Essai d'analyses de la conception paysanne de l'évolution, de la gestion et de l'utilité du Parc National de Mozogo-Gokoro (Cameroun) en vue de son aménagement. Int J. Biol. Chem. Sci., 7 (6): 2490-2503.

[17] Wanie I. S., 2020. Impact socio-économique et environnemental de l'exploitation de la végétation de Minawao dans le Département du Mayo-Tsanaga (ExtrêmeNord, Cameroun) Thèse de Doctorat/Ph.D., Faculte des Sciences, Université de N'Gaoundéré, 211p.

[18] Gaiwa D., Ibrahima A., Tchobsala, NoihaNoumi V. and ManNa D., 2019. Perception Paysanne Des Principales Contraintes Et Pratiques De La Production De La Gomme Arabique Dans $\mathrm{La}$ Province $\mathrm{Du}$ Chari-Baguirmi $\mathrm{Au}$ Tchad. International Multilingual Journal of Science and Technology, 4: 407-412.

[19] Halilou I., 2016. Impact de l'exploitation du bois-énergie sur les ressources ligneuses dans l'Arrondissement de Mokolo: cas de la localité de Gadala et sa périphérie. Mémoire de l'obtention du diplôme d'Ingénieur de Conception en Sciences Environnementales Université de Maroua, 73p.

[20] Nyasiri J., 2018. Anthropisation et dynamique spatiotemporelles des paysages forestiers de la falaise de Ngaoundéré (Adamaoua-Cameroun). Thèse doctorat $\mathrm{Ph} / \mathrm{D}$. Université de Ngaoundéré. 141p.

[21] Haiwa G., 2018. Impact de la déforestation sur la dynamique de la végétation en zone soudano sahélienne du Cameroun. Thèse de Doctorat/Ph.D., Université de Ngaoundéré. 259p.

[22] Idrissa I., Lawali S., Karim S., Marou B., Adagoye B. A. and Mahamane A., 2020. Perception communautaire de la dynamique de parcours naturels sahéliens des trente dernières années: cas de l'enclave pastorale de Dadaria (Maîné-Soroa, Diffa) au Niger. Afrique SCIENCE, 16 (5): 173-188.

[23] Laminou M. O., Morou B., Garba O. B. and Mahamane A., 2017. Usages Socio-economiques des espèces ligneuses au Sahel: Cas de Guidan Roumdji au Niger. European Scientific Journal, 13 (26): 1857-7881. 
[24] Sarr O., Diatta S., Gueye M., Ndiaye P. M., Guisse A. and Akpo L. E., 2013. Importance des ligneux fourragers dans un système agropastoral au Sénégal (Afrique de l'ouest). Revue Méd. Vét., 164 (1): 2-8.

[25] Ouya B. 2010. Conservation et utilisation durable de la biodiversité autour et dans les aires protégées du sud-est du Tchad: cas du parc de manda et de la forêt de Djoli-kra usages et risques, quelles stratégies pour la revalorisation et le développement local? Doctorat de géographie et aménagement de l'espace, Université Paul-Valéry. Montpellier-France, 296p.

[26] HOUNTONDJI Y. C. H., 2008. Dynamique environnementale en zones sahélienne et soudanienne de l'Afrique de l'Ouest: Analyse des modifications et évaluation de la dégradation du couvert végétal. Thèse de Doctorat, Université de Liège, 135 p.

[27] Boumba H. B. G. and Samba-Kimbata M. J., 2019. Analyse de l'impact des déterminants de dégradation du couvert végétal sur les composantes environnementales de la réserve de chasse de la Lefini (Congo). EDUCI), Revue de Géographie Tropicale et d'Environnement, (1): 75-89.

[28] Ballah S. R. and Ndoutorlengar M., 2017. Dynamique des ressources naturelles dans le Parc national de Manda: Cartographie et analyse pour le Développement durable. International Cartographic Association, 1-6.

[29] Anonymous, 2009. Le Rapport National sur la Diversité Biologique au Tchad; 4ème Edition, 81p.

[30] Ouya B., 2010. Restauration des terres dégradées de la lisière du Parc de Manda (Tchad) et développement local des pratiques et des réalités socio-spatiales contrastées «ISDA 2010, Montpellier: France», 1-8.

[31] FAO, 2015. Evaluation des ressources forestières mondiales, rapport national Tchad 90p.

[32] Ouaba P. A, Dan D E. C. and Pare S., 2014. Perception locale de la dynamique du peuplement ligneux des vingt dernières années au Sahel burkinabé. VertigO. La revue électronique en sciences de l'environnement, 14 (2), DOI: $10.4000 /$ vertigo. 15131 .

[33] Thiombiano D. N. E., Lamien N, Dibong D. S., Boussim I. J and Belem B., 2012. Le rôle des espèces ligneuses dans la gestion de la soudure alimentaire au Burkina Faso. Sécheresse, 23: 86-93. doi: 10.1684/sec.2012.0341.

[34] Anonymous, 2008. Ligneux du Sahel (1.0). CIRAD.

[35] Ouédraogo M., Ouédraogo D., Thiombiano T., Hien M. and Lykke A. M., 2013. Dépendance économique aux produits forestiers non ligneux: cas des ménages riverains des forêts de Boulon et de Koflandé au Sud-Ouest du Burkina Faso. Journal of Agriculture and Environment for International
Development, $\quad 107 \quad$ (1): $\quad 4572 . \quad$ DOI: http://dx.doi.org/10.12895/jaeid.20131.98

[36] Ilboudo A., Soulama S., Hien E. and Zombre P., 2020. Perceptions paysannes de la dégradation des ressources naturelles des bas-fonds en zone soudano-sahélienne: cas du sous bassin versant du Nakanbé-Dem au Burkina Faso. Int. J. Biol. Chem. Sci., 14 (3): 883-895.

[37] Guimbo D., Baragé I., and Douma M. S., 2012. Etudes préliminaires sur l'utilisation alimentaire des plantes spontanées dans les zones périphériques du parc $\mathrm{W}$ du Niger. Int. J. Biol. Chem. Sci., 6 (6): 4007-4017.

[38] Mahamane A., 2005. Études floristique, phytosociologique etphytogéographique de la végétation du Parc Régional du W du Niger. Thèse de doctorat en Sciences Agronomiques et Ingénierie Biologique, Faculté des Sciences, Université Libre de Bruxelles, 484p.

[39] Inoussa, M. M., 2011. Dynamique des forêts claires dans le parc national du $\mathrm{W}$ du Niger. Thèse de Doctorat en Ecologie Végétale, Faculté des Sciences et Techniques, Université Abdou Moumouni. 138p.

[40] Guigma, Y., Zerbo, P. and Millogo-Rasolodimby, J., 2012. Utilisation des espèces spontanées dans trois villages contigus du Sud du Burkina Faso. Tropicultura, 30 (4): 230-235.

[41] LE HOUEROU H. N., 1980. L'inventaire du potentiel fourrager des arbres et arbustes d'une région du sahel malien. Méthodes et premiers résultats. In: les fourrages ligneux en Afrique: Etat actuel des connaissances, Le Houerou H. N. (ed.), Addis Abeba, CIPEA, 481p.

[42] Saïdou. S., Ouoba P. A. and Da D. E. C., 2019. Extraction des PFNL et menace sur les espèces pourvoyeuses au Burkina Faso: Cas de Kokologho et Tenado dans la Région du CentreOuest. Rev. Ivoir. Sci. Technol., 33: 240-258.

[43] Busquet M. B., 2006. Des stratégies intégrées durables: savoir écologique traditionnel et gestion des espaces et des ressources. Vertigo 7 (2): 1-8.

[44] Smektala G., Peltier R., Sibelet N., Leroy M., Manlay R., Njiti C. F., Ntoupka M., Njiemoun A., Palou O. and Tapsou, 2005. Parcs agroforestiers sahéliens: de la conservation à l'aménagement. VertigO-la Revue Electronique en Sciences de l'Environnement, 6 (2).

[45] Bergonzini J., 2004. Changements climatiques, désertification, diversité biologique et forêts, SILVA, RIAT, 146p.

[46] Adjonou K., Bellefontaine R. and Kokou K., 2009. Les forêts claires du Parc National Oti-kéran au Nord Togo: structure, dynamique et impacts des modifications climatiques récentes. Sécheresse, 20 (1): 1-10. 